\title{
A laboratory model for plastic fragmentation in the turbulent ocean
}

\author{
Christophe Brouzet, ${ }^{1}$ Raphaël Guiné, ${ }^{1}$ Marie-Julie Dalbe, ${ }^{1}$ Benjamin Favier, ${ }^{1}$ \\ Nicolas Vandenberghe, ${ }^{1}$ Emmanuel Villermaux, ${ }^{1,2}$ and Gautier Verhille ${ }^{1, *}$ \\ ${ }^{1}$ Aix Marseille Univ, CNRS, Centrale Marseille, IRPHE - Marseille, France \\ ${ }^{2}$ Institut Universitaire de France, Paris, France
}

(Dated: March 25, 2021)

The fragmentation of solid objects in turbulence is of paramount importance in a large number of situations, especially for marine plastic pollution where small plastic debris are formed by the fragmentation of plastic litter under hydrodynamic forces. Up to now, investigations have focussed on the fragmentation of particle aggregates in turbulent flows. Here we study the fragmentation of a single deformable object that behaves elastically up to breakage, in the inertial range of turbulence. Using laboratory experiments with glass fibres as a model system, complemented by numerical simulations and theoretical analyses, we exhibit a comprehensive fragmentation scenario, further modeled by an evolution equation. Our results demonstrate that the fragmentation process is limited at small scales by a physical cut-off length originating from the fluid-structure interactions between the objects and the turbulence, and therefore independent of the brittleness of the fibres. This scenario leads to the accumulation of fragments with a typical length slightly longer than the cut-off scale, as smaller fragments are too short to be deformed and broken by the turbulence.

* gautier.verhille@irphe.univ-mrs.fr 


\section{INTRODUCTION}

Plastic is one of the most abundant man-made materials on Earth, with a current global production estimated at more than 300 million metric tons a year [1-4]. What makes its strength for a global use in our society, i.e. durability and low cost, leads to detrimental issues once it is released in natural environments $[2,3,5]$. Indeed, plastic debris are known to affect marine animals by ingestion or entanglement [6-8]. They also carry some persistent organic pollutants which can impact the entire food web by being transferred to water or animals [3, 6, 7, 9]. Moreover, it is established that plastic debris host a diverse microbial community that can travel across the globe on such durable substrates and impact the microbial loop in oceans $[9,10]$.

The global fragmentation process of large plastic items is described qualitatively in the literature [3, 5-8, 11-13]. Once in the ocean, these items are weathered by different mechanisms, mainly photo-degradation but also chemical, mechanical and bio-deterioration. The ensuing embrittlement of the plastic further leads to fragmentation into smaller pieces through wave action. However, as pointed out by Filella [5], this fragmentation process is mainly descriptive and a quantitative prediction of macroplastic fragmentation under relevant flow conditions is still missing [14]. Indeed, using results from studies of large plastic particle impacts on a wall [15, 16], Cózar et al. [17] suggested that the size distributions of floating plastic debris should exhibit a power law. Similar conclusions have been reached by Eriksen et al. [7] using conservative estimates of the fragmentation rates. Size distributions of floating plastic debris obtained from field measurements $[7,8,12,13,17-19]$ have recently gained attention by clearly exhibiting an accumulation of plastic debris around $1 \mathrm{~mm}$ and therefore a lack of small debris compared to the expected power law. To explain this gap, four main possible sinks for small microplastic debris have been proposed and discussed in the literature: shore deposition, nano-fragmentation, biofouling, and ingestion by marine organisms $[8,12,17]$. Despite slight discrepancies due to different locations where they have been measured [19], the size distributions however show that a general mechanism is at play during plastic fragmentation in the ocean.

Nevertheless, the fragmentation models mentioned above [15, 16] consider impact fragmentation [20] and are clearly disputable [5] for the fragmentation of brittle plastic debris in the ocean under wave action. For an in-depth understanding of the size distributions, it is therefore necessary to consider the influence of the turbulent environment [21] surrounding the particles, leading to their deformation and fragmentation. Therefore, we want to address the two following questions. What is the physical mechanism leading to such fragment size distributions ? Can we explain the main features of the distributions using arguments solely based on fluid-structure interactions ?

The fragmentation of solid objects by turbulent flows is poorly understood. Previous works have been mainly related to floc or aggregates fragmentation, whose cohesion is given by the interactions between the particules in the aggregates [22-28]. However, the mechanical properties of such structures are very different from those of a single and brittle object. To our knowledge, single particle fragmentation in a turbulent flow has been scarcely investigated and remains limited to polymer scission [29, 30]. In addition, most of these studies have been focused on objects smaller $[23-25,29,30]$ or comparable $[22,26]$ with the Kolmogorov length scale, the smallest scale of a turbulent flow. Since the Kolmogorov length scale near the ocean surface can be smaller than 1 mm [31, 32], macroplastic debris typically belong to the inertial range and interact with multiple scales of the turbulent flow.

In order to answer the questions discussed above, we study as a model system the fragmentation of flexible and brittle fibres in a laboratory turbulent flow, whose length $\ell$ lies within the inertial range of the turbulence. This study is particularly focused on the fragment length distributions obtained at long time scales, relevant for plastic fragmentation in oceans. We have chosen to focus on fibres for two reasons. First, as a fibre is a one-dimensional object, it is easier to describe mathematically [33] and both length and mass are conserved during the fragmentation process, contrary to two- or three-dimensional objects where only the mass is conserved [8]. Moreover, the behaviour of flexible fibres in the inertial range of a turbulent flow has been recently discussed [34-37]. In particular, one can define an elastic length for fibres in turbulence [35], which scales as [34]

$$
\ell_{e} \approx \frac{(E I)^{1 / 4}}{\left(\eta \rho_{f} \varepsilon\right)^{1 / 8}}
$$

where $E$ is the Young modulus of the fibre, $I=\pi d^{4} / 64$ its moment of inertia, $d$ its diameter, $\eta$ and $\rho_{f}$ the fluid dynamic viscosity and density, and $\varepsilon$ the mean turbulent dissipation rate. This length defines the transition between rigid $\left(\ell<\ell_{e}\right)$ and flexible fibres $\left(\ell>\ell_{e}\right)$, and corresponds to the correlation scale of the deformations for long flexible fibres [35]. In the remainder of the paper, all fibre lengths $\ell$ and curvatures $\kappa$ are made dimensionless using the elastic length, respectively as

$$
L \equiv \ell / \ell_{e} \quad \text { and } \quad K \equiv \kappa \ell_{e}
$$

Here, we show that the elastic length plays a key role in the fragmentation process of flexible and brittle fibres in the inertial range of a turbulent flow. Indeed, by monitoring the deformation along the fibres, we demonstrate that it 
controls the fragmentation process and leads to specific fragment length distributions, with a minimal fragment length close to the elastic length. These fragment length distributions are first observed in both experiments and numerical simulations. We then explicit a comprehensive fragmentation scenario, modeled theoretically by an evolution equation. This equation is first solved analytically in a simplified version, yet able to recover the main features of the fragment length distributions observed experimentally and numerically. We then solve numerically this evolution equation and compare quantitatively the fragment length distributions obtained in the experiments, the numerical simulations and the model. Finally, by evaluating the elastic length for typical plastic debris in the ocean, we demonstrate that the fragmentation scenario described here is fully compatible with the observed fragment length distributions of plastic debris in the ocean. Our model laboratory experiments, numerical simulations and theoretical derivations therefore provide a unified framework for understanding the fragmentation of plastic in the turbulent ocean, going beyond heuristic models currently used in oceanography.

\section{LABORATORY SETUPS}

\section{A. Experiments}

\section{Turbulent flow}

Laboratory experiments are performed in a turbulent von Kármán flow $[28,34,35]$ generated in a cubic tank by two contra-rotating propellers, as shown in Fig. 1(a). This well-known flow has been extensively used in turbulence studies [38] and is here considered as a model turbulent flow, despite that the fluid and particle dynamics close to the free-surface of the ocean are much more complex [21,39-42]. The tank is the same than the one used in a previous work of our group [28], without the grids close to the propellers. It is a transparent acrylic cube with a typical size of $200 \mathrm{~mm}$, containing a volume $V$ of approximately 8 litres of liquid. Each propeller has a radius $R=86 \mathrm{~mm}$, fitted with 8 straight blades with a height of $5 \mathrm{~mm}$. The two propellers rotate at the same frequency $f=15 \mathrm{~Hz}$ in a classical contra-rotating configuration $[28,34,35,38,43]$. Two fluids have been used in the tank in order to vary the dynamic viscosity $\eta$ : pure water $(\eta=1 \mathrm{mPa} . \mathrm{s})$ and a mixture of water and $30 \% \mathrm{Ucon}$ oil $(\eta \approx 100 \mathrm{mPa} . \mathrm{s})$. The turbulence generated by a von Kármán flow is known to be non homogeneous and more intense close to the propellers [38]. The global turbulent dissipation rate $\varepsilon$ is about $50 \mathrm{~m}^{2} / \mathrm{s}^{3}$, independently of the fluid used as the forcing is inertial and not viscous. The corresponding Kolmogorov length scale $\eta_{K}$ and time scale $\tau_{K}$, together with the Reynolds number Re of the flow are given in Table I. The integral scale $\ell_{I}$ is about $30 \mathrm{~mm}$ while the integral time scale $T_{I} \approx 1 / f$ is about $67 \mathrm{~ms}$ for all experiments. Experiments have always been performed in a dilute regime [44], i.e. with a number of initial fibres $N_{0}$ such that

$$
N_{0}<\frac{V}{\ell_{0}^{3}}
$$

where $V$ is the volume of the tank and $\ell_{0}$ the initial fibre length. This means that the fibres do not interact with each other and do not modulate the intensity or the properties of the turbulent flow.

\section{Flexible fibres}

The fibres used in the laboratory experiments are glass fibres. They all originate from the same batch and have been given by Owens Corning company. The diameter of the fibres has been measured by taking several images of different fibres using a microscope, as shown in Fig. 1(b). All the measured fibres have shown a diameter $d=20 \mu \mathrm{m}$ with a very small dispersion, as specified by the manufacturer. The Young modulus $E$ has been obtained by measuring the deflection $\delta$ from the horizontality for fibres placed at the edge of an horizontal plate (see Appendix A 1). The measured value of $E=41 \mathrm{GPa}$ corresponds to the order of magnitude expected for glass [45, 46]. These fibre

TABLE I. Fluids, flow characteristics, elastic lengths and breaking parameters for the experiments performed in this study.

\begin{tabular}{cccccc}
\hline \hline Fluid & $\eta_{K} \equiv\left(\nu^{3} / \varepsilon\right)^{1 / 4}$ & $\tau_{K} \equiv(\nu / \varepsilon)^{1 / 2}$ & $\mathrm{Re}=2 \pi R^{2} f / \nu$ & $\ell_{e}$ & $K_{B} \equiv \kappa_{B} \ell_{e}$ \\
\hline Water & $12 \mu \mathrm{m}$ & $0.14 \mathrm{~ms}$ & $7 \times 10^{5}$ & $2.6 \mathrm{~mm}$ & $4.3 \pm 0.9 / 2.1 \pm 0.5$ \\
Water + Ucon oil mixture & $375 \mu \mathrm{m}$ & $1.4 \mathrm{~ms}$ & $7 \times 10^{3}$ & $1.5 \mathrm{~mm}$ & $2.5 \pm 0.5 / 1.2 \pm 0.3$ \\
\hline \hline
\end{tabular}




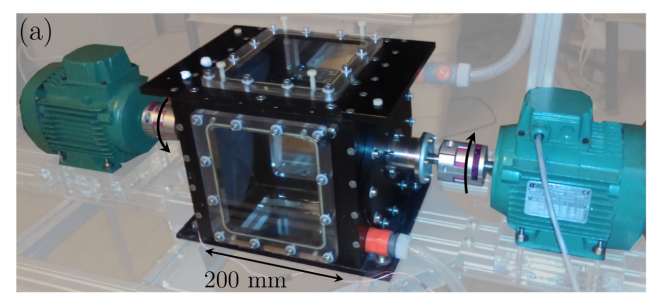

(d)

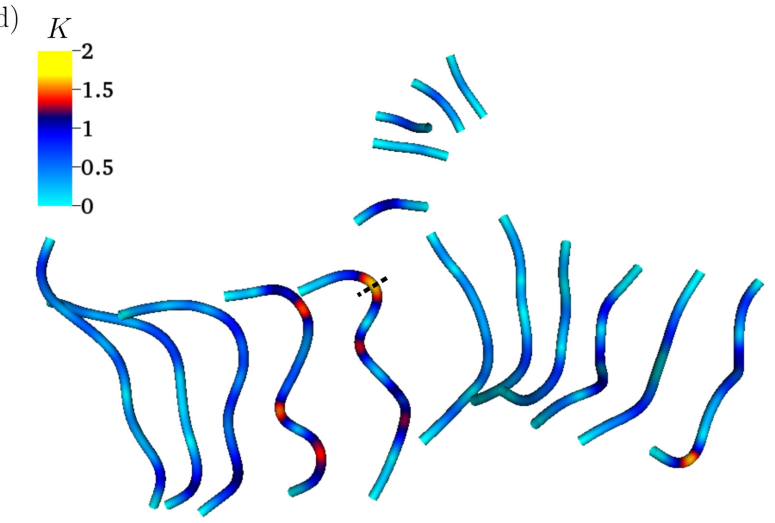

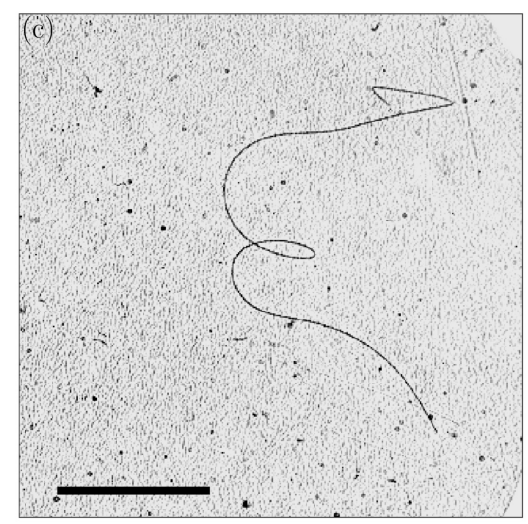

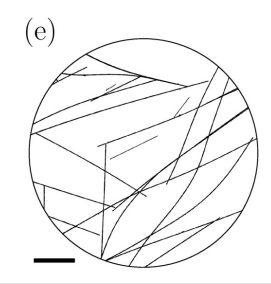

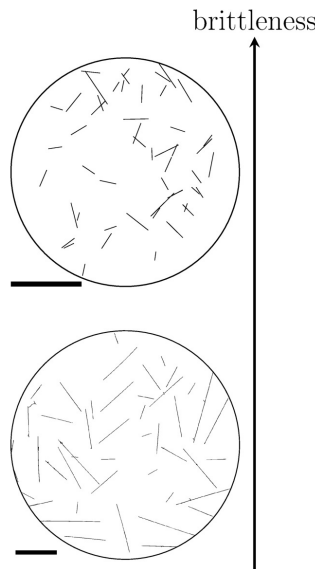
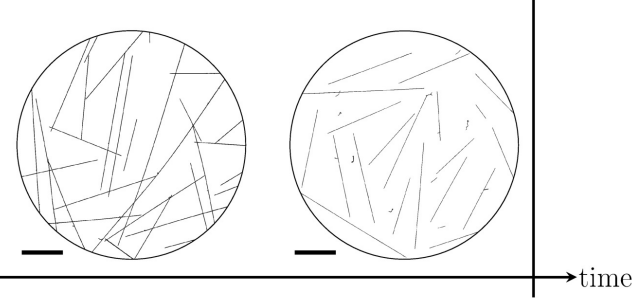

FIG. 1. Plastic fragmentation: from oceans to the laboratory. (a) Picture of the von Kármán turbulent flow used in the experimental setup and described in section II A 1. The black arrows indicate the direction of rotation of the two motors. (b) Glass fibre observed under a microscope for diameter measurement. (c) 2D image of a glass fibre deformed by the experimental turbulent flow observed using a schlieren technique. (d) Breakup event obtained in the numerical simulations that are described in section II B. The event is illustrated using the superimposition of different time steps, with time going from left to right. The colors correspond to the local curvature $K$ and the location of the upcoming event on the fibre is shown by a dashed dotted line. More details on the fragmentation mechanism are given in section II C. (e) Random sets of fibre fragments collected in the experimental turbulent flow (see section IID), for different times and breaking parameters $K_{B}$. The fibre fragments are used to obtain fragment length distributions, shown in Fig. 2 and discussed in section III A. The scale bars in panels (c) and (e) all indicate $10 \mathrm{~mm}$, while the diameter of the circles in panel (e) is equivalent to $20 \ell_{e}$ for all cases.

characteristics allow us to estimate the elastic length in the two different fluids used. As shown in Table I, the elastic length $\ell_{e}$ belongs to the millimeter range.

The glass fibres are placed in the turbulent flow with an initial length $\ell_{0}=50$ or $80 \mathrm{~mm}$. In this range, we see no influence on the fragmentation process on long time scales or for short fragments, providing that $L_{0} \equiv \ell_{0} / \ell_{e}$ is significantly larger than 1 . More details about the effect of the initial length on the fragmentation process are given in Appendix B. With the typical length scales used in the laboratory experiments and given above, it is important to note two main points. First, the fibres have a typical length much longer than the Kolmogorov scale and a diameter of the same order or smaller (depending on the fluid used). This means that the fibres are effectively in the inertial range of the laboratory turbulent flow, with a forcing which is mainly viscous. Second, the initial fibre length is also much larger than the elastic length. This therefore shows that the fibres are indeed deformed by the turbulence (for more details on fibre deformation, see [35]). They are therefore considered as flexible in the following of the paper. An example of a glass fibre deformed by the experimental flow is shown in Fig. 1(c).

In addition, note that the gravity has a negligible influence on the fibre dynamics in the experiments, despite the large density difference between the glass fibres and the fluid. Indeed, the typical settling velocity can be estimated to be about 2 to $5 \times 10^{-2} \mathrm{~m} / \mathrm{s}$ depending on the fibre length [47], while the typical root mean square (rms) velocity $U$ of the flow is of the order of $1 \mathrm{~m} / \mathrm{s}$, and therefore much larger than the settling velocity of the fibre. 


\section{B. Numerical simulations}

The laboratory experiments are complemented by numerical simulations, using a numerical setup described recently [35]. In these idealised simulations, the equation for a 1D inextensible elastic rod is solved

$$
\rho_{p} S \frac{\partial^{2} \vec{r}}{\partial t^{2}}-\frac{\partial}{\partial s}\left(T \frac{\partial \vec{r}}{\partial s}\right)+E I \frac{\partial^{4} \vec{r}}{\partial s^{4}}=\vec{\xi}
$$

where $\rho_{p}$ and $S$ are respectively the fibre density and cross-section, $\vec{r}$ the position of the fibre centre line, $s$ the curvilinear coordinate, $T$ the tension term ensuring the inextensibility, and $\vec{\xi}$ the hydrodynamic forcing term. As the particle Reynolds number is small and by ignoring the anisotropic nature of the viscous drag for slender bodies [48], the forcing term can be approximated by $\vec{\xi} \approx 4 \pi \eta \vec{u}_{\text {slip }} / \ln (2 \ell / d)$, where $\vec{u}_{s l i p}=\vec{u}-\partial \vec{r} / \partial t$ is the slip velocity between the fluid and the fibre. Kinematic Simulations [49] are used to generate the turbulent flow field $\vec{u}$, as a superposition of incompressible random Fourier modes. By rescaling space with the integral length scale of the turbulent flow $\ell_{I}$, velocity with the rms velocity $U$, time with the integral time scale $T_{I}=\ell_{I} / U$, tension $T$ with $\rho_{p} S U^{2}$, and bending rigidity $E I$ with $\rho_{p} S U^{2} \ell_{I}^{2}$, Eq. (4) can be made dimensionless and depends on two dimensionless parameters, the bending rigidity

$$
B=\frac{E I}{\rho_{p} S U^{2} \ell_{I}^{2}}
$$

and the Stokes number

$$
\mathrm{St}=\frac{d^{2}}{16 \nu} \frac{\rho_{p}}{\rho_{f}} \frac{U}{\ell_{I}} \ln (2 \ell / d)
$$

These two parameters have been set to $B=10^{-2}$ and $\mathrm{St}=2 \times 10^{-2}$. The value for the Stokes number is similar to the one in the experimental setup. This means that the fibres tend to follow the flow and do not behave as inertial particles, even if particle density is larger than the fluid density. The bending rigidity is smaller than in the experiments and the consequences of this choice are discussed in the following section. With these parameters, note that the elastic length in the simulations is about $\ell_{I} / 10$ while the initial length $\ell_{0}$ of the fibres is set to the integral scale $\ell_{I}$. This means that the simulations also focus on fibres in the inertial range of turbulence and that the fibres are flexible and deformed by the turbulence, as shown in Fig. 1(d).

\section{Fragmentation mechanism}

As marine plastic items are weathered by oceanic conditions and become brittle $[3,5,6]$, our laboratory model focuses on the fragmentation of brittle objects in turbulence. This has therefore motivated the choice of the material for the fibres used in this study, as glass is known to be brittle [50]. For a brittle object, the deformation at break remains in the elastic regime and there is no significative permanent deformation before breaking. As a consequence, fragmentation is here assumed to occur locally when the local curvature $\kappa$ of the fibre, elastically deformed by the turbulence (see Figs. 1(c) and (d)), reaches a critical breaking curvature $\kappa_{B}$. We therefore define a dimensionless control parameter of the fragmentation process by balancing the critical curvature with the typical length scale of the deformation: $K_{B} \equiv \kappa_{B} \ell_{e}$. Even though the fragmentation process of brittle materials and especially of glass [51] is more complex [52], the breaking parameter $K_{B}$ directly quantifies the brittleness of a given fibre in the flow.

Experimentally, the critical curvature $\kappa_{B}$ where fragmentation occurs is delicate to define for brittle materials [51, 52]. However, it can be estimated using the tensile strength of the fibres $\sigma_{B}$ as

$$
\kappa_{B} d=2 \frac{\sigma_{B}}{E} .
$$

The tensile strength of glass fibres is governed by defects located randomly on the fibres $[45,46,50]$ and is naturally broadly dispersed (see Appendix A 2). As a consequence, it is possible to increase the brittleness of the fibres by using a heat-treatment on the fibres. Indeed, this leads to the growth of pre-existing surface defects and/or to the creation of new ones [46], thus reducing the tensile strength. In our case, raw fibres have been placed in an oven at $550^{\circ} \mathrm{C}$ during $60 \mathrm{~min}$, with a heating phase from ambient temperature to $550^{\circ} \mathrm{C}$ of about $30 \mathrm{~min}$. Such heat-treatment reduces the tensile strength of the fibres by a factor 2 on average (see Appendix A 2), but does not affect their Young modulus (see Appendix A 1), as reported earlier [46]. This means that the Young modulus value of $E=41 \mathrm{GPa}$ 
can always be used in this study for the calculation of the elastic length $\ell_{e}$ (see Eq. (1)) or for the estimation of the critical curvature $\kappa_{B}$ (see Eq. (7)), whether the fibres have been heat-treated or not. By decreasing the elastic length $\ell_{e}$ through changes in the viscosity of the fluid (see Eq. (1)) and by reducing the tensile strength of the fibres, and therefore their critical curvature $\kappa_{B}$ (see Eq. (7), through heat-treatment [46], we have explored four different breaking parameters $K_{B}$ in the experiments, as summed up in Table I.

Moreover, each breakup event is assumed to produce only two fragments. Classically for impact fragmentation of brittle fibres [20], a breakup event leads to a relaxation bending wave along the fibre, inducing higher curvatures elsewhere on the fibre and causing other breakup events. This phenomenon produces broader fragment length distributions, including the existence of tiny fragments called fines. However, in our experiments and simulations, the Stokes number is much smaller than 1. So inertia is negligible and relaxation bending waves are rapidly damped. Therefore, a breakup event always leads to the production of only two fragments, contrary to the fragmentation in air of a spaghetti for example [20]. Note that this low inertia also minimizes the risk of direct impact with the propellers, because the fibres tend to follow the flow.

Such fragmentation mechanism has been implemented in the numerical simulations where the breaking parameter $K_{B}$ is comparable to that of the experiments. For numerical convenience, the bending rigidity $B$ (see Eq. (5)) is about one order of magnitude smaller than in the experiments, in order to have more flexible fibres and therefore fragmentation events that are more probable. Indeed, as shown previously [35], the maximum curvature along the fibre decreases with the bending rigidity $B$. For a given $K_{B}$, a lower bending rigidity allows us to gather a large number of fragmentation events. This is one possible explanation for the different orders of magnitude for the time evolution of the fragment length distributions in the experiments $\left(t / T_{I} \sim 10^{5}\right)$ and in the numerical simulations $\left(t / T_{I} \sim 10^{2}\right)$, even though the breaking parameters $K_{B}$ are similar (see Section III A).

In the simulations, each fibre is released individually and initially straight, with a random orientation and position in the flow. We then track the evolution of the fibre curvature with time following Eq. (4): when the local curvature reaches the critical threshold, the fibre is split into two fragments (see Fig. 1(c)). Each fragment is then evolved independently until it breaks again or until it does not experience any more breakup event during an arbitrary time after which we assume that no fragmentation will ever occur, fixed to $400 T_{I}$. This process is repeated until all fragments have reached equilibrium, i.e. when they do not fragment after $400 T_{I}$. We checked that the sum of the fragment lengths is always equal to the original fibre length. We then repeat this sequence for the same parameters using a different random initialisation.

\section{Fragment length distributions measurements}

The fragment length distributions presented in this study contain more than 600 fragments for each experiment and about $10^{4}$ fragments for the numerical simulations. Note that the unbroken fibres of length $L_{0}=\ell_{0} / \ell_{e}$ are excluded from the statistics. Experimentally, a known number of fibres with an initial length $L_{0} \equiv \ell_{0} / \ell_{e} \gg 1$ (see Appendix B) is introduced in the tank and the flow is then driven for a given time. As the fibres are transparent and have a small diameter, fragment lengths cannot be directly measured in the flow using the available optical systems. All the fragments are therefore collected directly in the tank one by one with tweezers and measured ex situ. Since long fragments are easier to collect than shorter ones, we paid attention to collect everything that has been introduced in the tank initially. By adding the lengths of all collected fragments, we recover the total length introduced in the tank, with a maximum error of $5 \%$. This is sufficient to ensure that there is no oversampling of long fibres compared to shorter ones. Furthermore, as expected due to the small inertia of the fibres, this allows us to say that there is probably no fine, i.e. tiny fragment, created during the fragmentation process. Fig. 1(e) shows examples of random sets of fibres fragments collected in the experiments at different times and for different brittlenesses (i.e. different breaking parameters $K_{B}$ ). It qualitatively shows that typical fragment length reduces with time as expected, and that the brittler fibres break faster.

\section{EXPERIMENTAL AND NUMERICAL RESULTS}

\section{A. Fragment length distributions}

The evolutions of the experimental fragment length distributions as a function of time $t / T_{I}$ and breaking parameter $K_{B}$, qualitatively illustrated in Fig. 1(d), are shown in Figs. 2(a) and (b). As time increases or $K_{B}$ decreases, long fragments disappear by creating shorter ones, thus leading to their accumulation. However, this process seems to slow down and it is necessary to wait for a long time to capture a significative evolution in the length distributions in Fig. 2(a). These laboratory length distributions all exhibit a minimal fragment length, a maximum of abundance close 

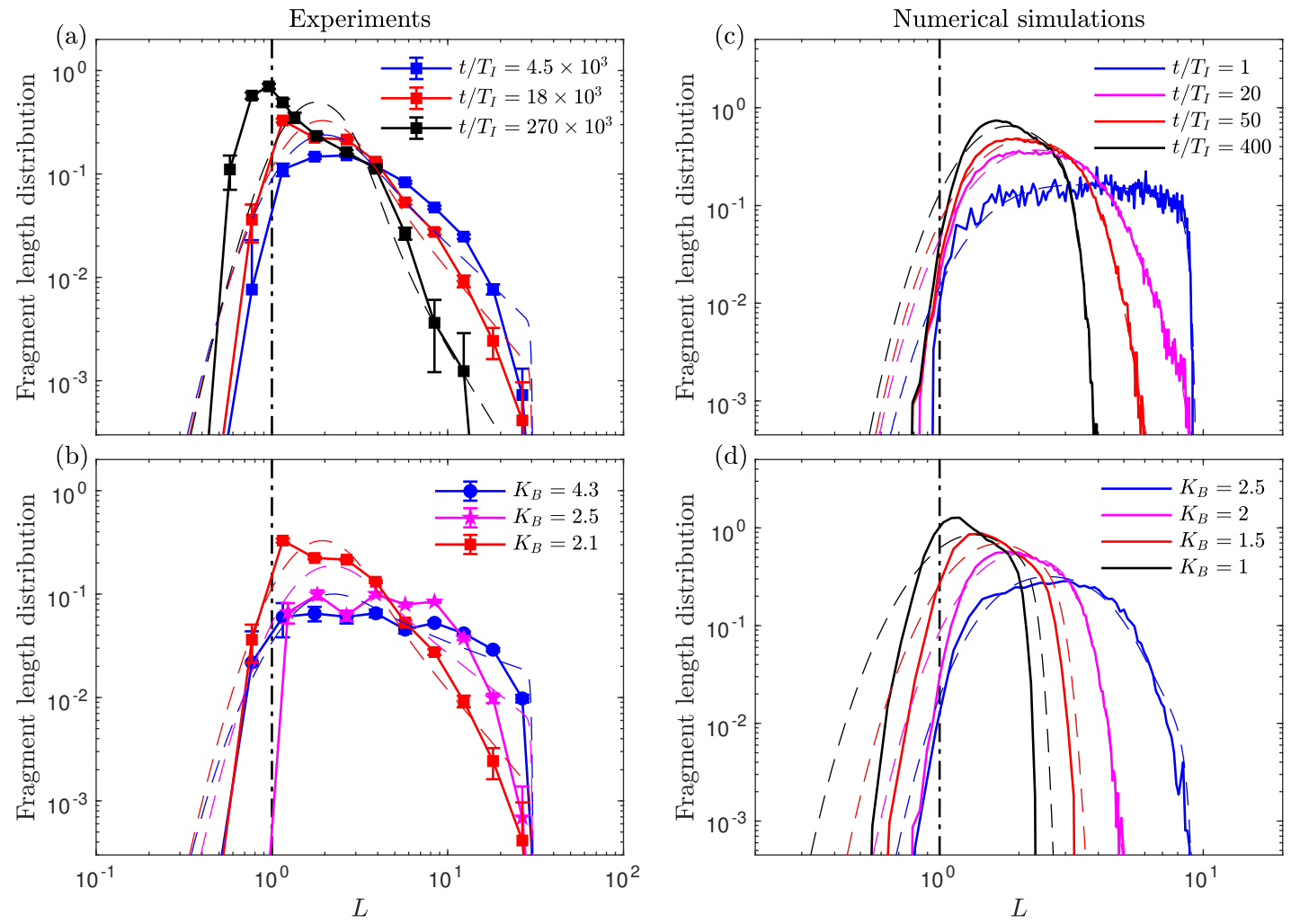

FIG. 2. Experimental (left) and numerical (right) fragment length distributions with $L \equiv \ell / \ell_{e}$. Top line shows the time evolution for a fixed breaking parameter $K_{B}=2.1$ in experiments (a) and $K_{B}=2$ in the simulations (c). Bottom line corresponds to the evolution as a function of breaking parameter for a fixed time $t / T_{I}=18 \times 10^{3}$ in experiments (b) and $t / T_{I}=100$ in the simulations (d). With corresponding colors, the thin dashed lines represent the model discussed further in the text (see Section IV C). The vertical dashed dotted lines indicate $L=1$ in all panels and the error bars represent \pm 4 fragments for the experiments.

to this minimal fragment length and a relatively long tail, leading to an asymmetry. Such characteristics ressemble those of the plastic size distribution obtained in oceans [17-19]. In the laboratory, these characteristics are however correlated to the elastic length $\ell_{e}$, as the minimal fragment length is slightly lower than $L=1$ and the maximum is located around $L^{\star} \approx 1-3$.

The fragment length distributions obtained using the numerical simulations are shown in Figs. 2(c) and (d), for different times $t / T_{I}$ and breaking parameters $K_{B}$ respectively. They clearly exhibit the same evolutions and characteristics as the experimental fragment length distributions, showing that the fragmentation mechanism implemented in the simulations is fully sufficient to recover a similar fragmentation scenario. We recall that the typical timescales involved in the numerical model are much shorter than in the experiments. This is a consequence of the smaller bending rigidity used in the numerics to reduce the numerical cost by speeding up the fragment process. The fact that the fragment distributions are similar in both cases despite this difference indicates the robustness of the underlying mechanism. Moreover, the simulations allow us to extract relevant statistics on the fibre deformations in order to identify the physical ingredients of the fragmentation process leading to such length distributions. Indeed, as fragmentation occurs when the fibres are significantly deformed by the turbulence, the fragmentation scenario directly depends on the evolution of the fibre deformation statistics with the fibre length.

\section{B. Deformation statistics}

This section is based on the numerical results only. The local curvature along the fibre, averaged over time and realisations, $\langle K\rangle \equiv\langle\kappa\rangle \ell_{e}$ is shown in Fig. 3(a) for different fibre lengths. For long fibres, $\langle K\rangle$ is uniform on a plateau near the centre of the fibre with a maximum defined as $\langle K\rangle^{\star}$, and drops to 0 on the two tips due to the free end boundary condition, with a "boundary layer" thickness fixed by the elastic length [35]. For shorter fibres less likely 

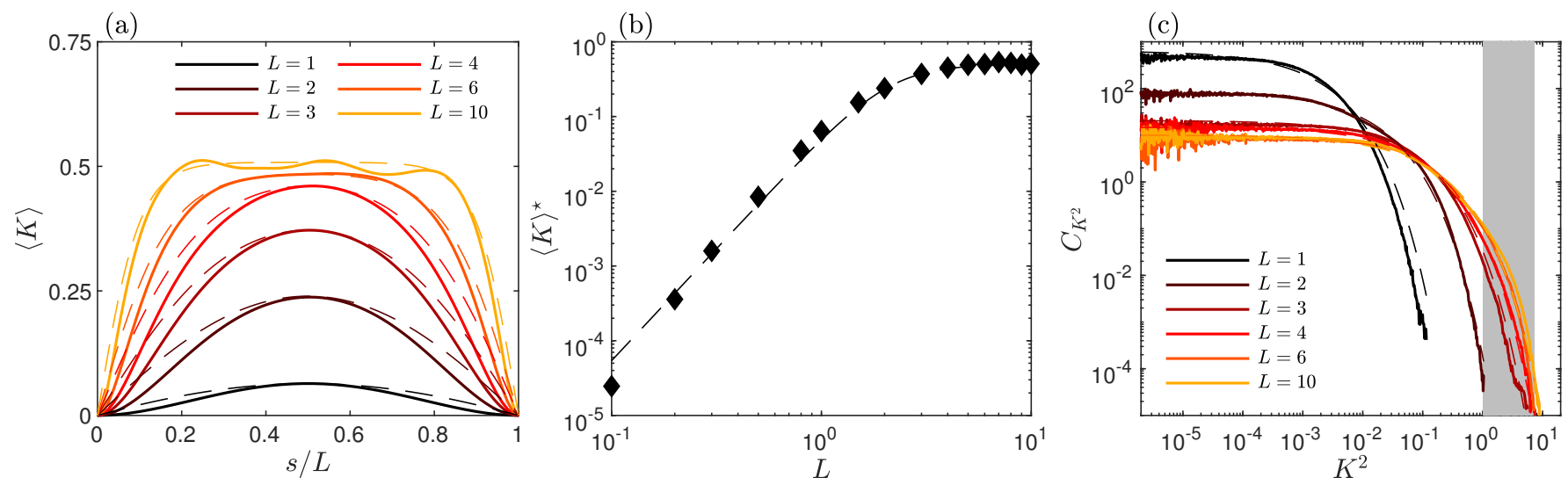

FIG. 3. Deformation statistics. (a) Local curvature $\langle K\rangle$ as a function of the curvilinear coordinate $s / L$ along the fibre (solid lines) and its approximation from Eq. (8) (thin dashed lines) for different fibre lengths. The notation \langle\rangle denotes an average over time and realisations. (b) Maximum amplitude $\langle K\rangle^{\star}$ of the curvature $\langle K\rangle$ along the fibre as a function of length $L$. The symbols represent the data from the numerical simulations while the dashed line correspond to the fit using Eq. (11). (c) Curvature statistics $C_{K^{2}}$ obtained from the numerical simulations (solid lines) and their fit (thin dashed lines) by Eq. (12) for different fibre lengths $L$. The gray region indicates the range of critical curvatures $K_{B}^{2}$ explored numerically.

to deform, the plateau for $\langle K\rangle$ in the centre is narrower and its amplitude $\langle K\rangle^{\star}$ decreases with the fibre length. The curvature $\langle K\rangle$ along the fibre can be approximated by the empirical formula

$$
\langle K\rangle(s, L)=\langle K\rangle^{\star} \frac{\tanh (s)+\tanh (L-s)-\tanh (L)}{2 \tanh (L / 2)-\tanh (L)},
$$

where $s$ is the curvilinear coordinate from one tip to the other. The variation of the maximum amplitude $\langle K\rangle^{\star}$ as a function of fibre length $L$ is shown in Fig. 3(b). While the maximum amplitude $\langle K\rangle^{\star}$ on the plateau is constant for long fibres, it behaves as $\langle K\rangle^{\star} \sim L^{3}$ for fibres shorter than $L=1$, i.e. fibres shorter than the elastic length $\ell_{e}$. Using similar power argument than the one used for the definition of the elastic length scale [34], the scaling for short fibre lengths can be understood by balancing the turbulent and bending powers

$$
\rho_{f} \varepsilon \ell^{3} \sim \frac{E I \kappa^{2} \ell}{\tau_{v}} .
$$

Here, the bending power is expressed as the curvature energy $E I \kappa^{2}$ integrated over the fibre length $\ell$ and divided by the relaxation time $\tau_{v} \sim \eta \ell^{4} /(E I)$. This power balance leads to a scaling for the typical curvature of fibres shorter than $L=1$ as

$$
\kappa \sim \frac{\left(\rho_{f} \eta \varepsilon\right)^{1 / 2}}{E I} \ell^{3} .
$$

The dependence of $\langle K\rangle^{\star}$ as a function of fibre length $L$ can therefore be fitted by

$$
\langle K\rangle^{\star}(L)=\frac{1}{\beta_{0} L^{-3}+\beta_{\infty}},
$$

with $\beta_{0} \approx 18.5$ and $\beta_{\infty}=2$, as shown in Fig. $3(\mathrm{~b})$. Note that $1 / \beta_{\infty}$ sets the maximum amplitude of the curvature $\langle K\rangle$ for the longest fibres and depends on the bending rigidity of the fibre given in Eq. (5) [35].

The breaking probability is given by the probability of the local curvature $K$ to be greater than the critical curvature $K_{B}$. It can then be deduced from the distribution of the local curvature. By simplicity, we will consider in the following the distribution of $K^{2}$ which presents a plateau at small value, as shown in Fig. 3(c). The distributions have been computed on the plateau for fibres longer than $L=1$ and around the middle of the fibre for shorter fibres. They can be empirically well fitted by the distribution

$$
C_{K^{2}}(x) \propto \frac{1}{1+\exp \left(\beta(L)\left(x^{1 / 2}+1\right)\right)},
$$


shown by thin dashed lines in Fig. 3(c). Here $\beta$ is the only fitting parameter, connected to the mean curvature $\bar{K}$ as

$$
\bar{K} \equiv \int_{0}^{+\infty} x^{1 / 2} C_{K^{2}}(x) \mathrm{d} x=\frac{2}{\beta(L)} .
$$

As $\bar{K} \sim\langle K\rangle^{\star}$ because the curvature distributions in Fig. 3(c) corresponds to the fibre curvilinear coordinates $s$ where the curvature $\langle K\rangle$ is maximum, $2 / \beta$ follows well the fit proposed in Eq. (11). The parameter $\beta$ can be seen as the inverse of an effective temperature in an analogy with polymer physics.

\section{Fragmentation statistics}

The deformation statistics are now used to obtain some key features of the fragmentation process, i.e. how often the fibres break and where they break the most. To evaluate the breaking probability, noted $p(L)$, one directly uses the curvature distributions obtained in the numerical simulations, shown in Fig. 3(c). Obviously, when $K_{B}$ is larger than the most extreme curvature events, i.e. beyond the grey zone in Fig. 3(c), there is no fragmentation. When $K_{B}$ is in the range corresponding to the tails of the curvature statistics for fibres much longer than $L=1$, i.e. in the grey zone in Fig. 3(c), fragmentation starts to occur but is not so frequent. In this study, we mainly focus on the fragmentation process in this regime. When $K_{B}$ is smaller than 1, i.e. before the grey zone in Fig. 3(c), fragmentation occurs very easily and fibres shorter than $L=1$, which are almost rigid, may nevertheless break. However, this last regime remains very difficult to observe experimentally or numerically, due to the very large number of short fragments it generates, and is only briefly discussed further in this study. The probability $p_{L}\left(K_{B}\right)$ that the local curvature $K$ exceeds $K_{B}$ at a given position on a fibre of length $L$ and per unit time is given by

$$
p_{L}\left(K_{B}\right)=\int_{K_{B}^{2}}^{+\infty} C_{K^{2}}(x) \mathrm{d} x .
$$

As the fragmentation results from local curvature events that are independent and as the correlation scale of the deformations for long flexible fibres is the elastic length [35], one can then consider that the fibre is composed of $L$ independent pieces of unit length (i.e. $\ell_{e}$ in dimensional scale). The total breaking probability $p(L)$ is therefore given by the combination of the breaking probability of the individual pieces

$$
p\left(K_{B}, L\right)=1-\left(1-p_{L}\left(K_{B}\right)\right)^{L},
$$

and can be approximated by $p\left(K_{B}, L\right) \approx p_{L}\left(K_{B}\right) L$ for finite $L$ and small $p_{L}$. This means that, for long fibres, the breaking probability is extensive with the length of the fibre. The breaking probability $p\left(K_{B}, L\right)$ is shown with solid lines in Fig. 4(a), as a function of fibre length $L$ and for the four breaking parameters $K_{B}$ used in the numerical simulations. For long fibres, the probability exhibits the scaling $p(L) \propto L$ as it is extensive (see dashed lines in

(a)

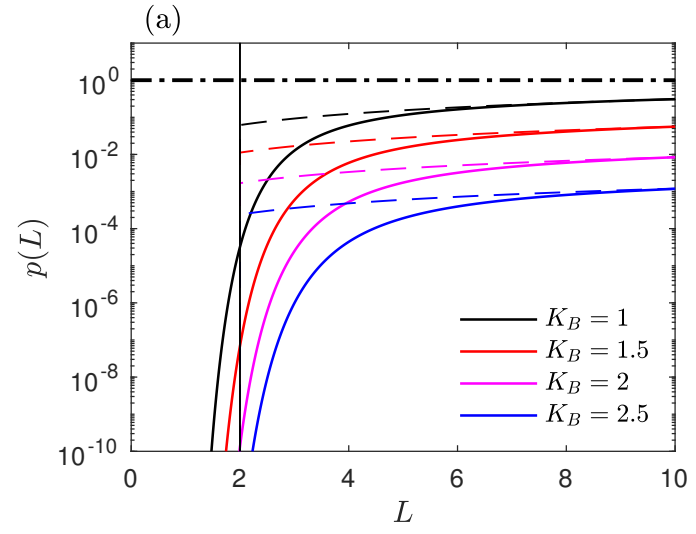

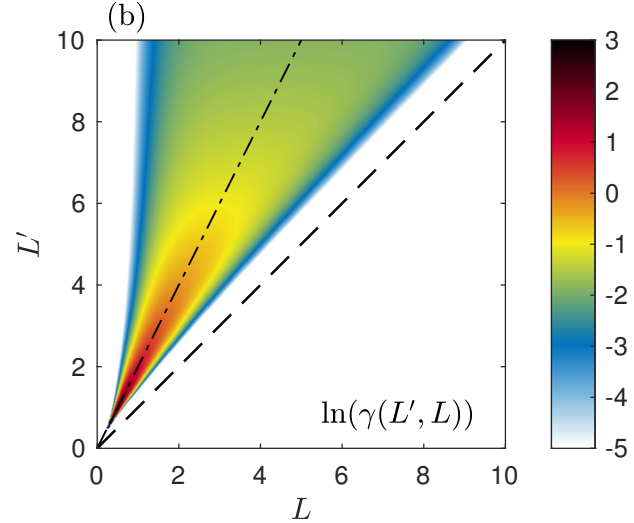

FIG. 4. Fragmentation statistics. (a) Breaking probability $p$ as a function of fibre length $L$ (solid lines) and its model from Eq. (19) (dashed lines) for different breaking parameters. The horizontal dashed dotted line indicates $p \equiv 1$ and the vertical thin solid line shows $L=L_{c} \equiv 2$. (b) Logarithm of the breaking location probability $\gamma$ as a function of fibre lengths $L$ and $L^{\prime}$ for $K_{B}=2$. The dashed line shows $L=L^{\prime}$ while the dashed dotted one indicates $L=L^{\prime} / 2$. 
Fig. 4(a)), but $p(L)$ drops drastically for fibres shorter than $L \approx 3$. Indeed, as shown in Fig. 3(c), these fibres have almost no curvature events in the grey zone representing the critical curvatures $K_{B}$ considered here.

As each fragmentation event leads to only two pieces, the location where such event occurs on the fibre influences drastically the fragment length distribution. The breaking location probability $\gamma\left(L^{\prime}, L\right)$ represents the statistics of the breaking location along the fibre and gives the probability to obtain a fibre of length $L$ by fragmentation of a fibre of length $L^{\prime}$. It can be estimated by examining the curvature $\langle K\rangle$ along the fibre, shown in Fig. 3(a). As the curvature $\langle K\rangle$ is maximal in the centre of the fibre and goes to 0 at the two undeformed tips, we assume that the higher this curvature at a given location on the fibre, the larger the curvature fluctuations, and the more likely a fibre is to break at that location. Because the curvature statistics $C_{K^{2}}$ depend on the fibre length $L$, the mean curvature $\bar{K}$ (see Eq. (13)) and the breaking probability $p_{L}$ (see Eq. (14)) are also functions of the same fibre length $L$. As both of these functions are monotonic with $L$, one can therefore establish a bijection between the two, i.e. obtain the function $p_{L}(\bar{K})$. This allows us to assign a given local breaking probability $p_{L}(\langle K\rangle)$ to the fibre curvilinear coordinates $s$ with a given curvature $\langle K\rangle$ (see Eq. (8)) through the correspondance $\langle K\rangle=\bar{K}$. After a proper normalisation to verify that for all $L^{\prime}$

$$
\int_{0}^{L^{\prime}} \gamma\left(L^{\prime}, L\right) \mathrm{d} L=1,
$$

we finally obtain a numerical evaluation of $\gamma\left(L^{\prime}, L\right)$, shown in Fig. 4(b). As expected, it indicates for long fibres that a breaking event has the same probability to occur everywhere on the fibre except on the two tips where the probability is much lower, while short fibres have the highest probability to break in the center.

\section{Fragmentation scenario}

Figure 4 therefore shows that fragmentation is far more probable for long fibres than for short ones, and mainly occurs in the plateau where the curvature $\langle K\rangle$ is maximum but not close to the tips. It now allows us to establish a comprehensive scenario for the fragment process when $K_{B} \geq 1$. Early in the process, Fig. 4(b) shows that long fibres break everywhere except on the edges, with an extensive breaking probability $(p \propto L)$, as visible in Fig. 4(a). This initially leads to flat length distributions depleted on the two sides, and therefore without fragments shorter than $L=1$. As fibre length is decreased through the fragmentation process, the fibres are less likely to deform and to break. This leads to a drastic reduction of the breaking probability for fibres shorter than $L \approx 3$ (see Fig. 4(a)), responsible for the slow-down of the fragmentation process. As a consequence, short fibres break less frequently and mainly at the centre in two equal pieces (see Fig. 4(b)), until $L \approx 2$ where fibres do not fragment further as their typical maximal curvature does not reach the critical one (see Fig. 3(c)). This maintains a minimal fragment length around $L=1$ and leads to the accumulation of fragments slightly longer than the minimal length, with a length distribution peaking at $L^{\star} \approx 1-3$. This fragmentation scenario shows that the elastic length $\ell_{e}$, the deformation length scale of the fibre, controls the fragment length distributions by acting as a cut-off length. It is important to note that this length does not depend on the brittleness of the fibre, as shown by Eq. (1). In that regime, the brittleness of the fibre, i.e. the breaking parameter $K_{B}$, is shown to non-linearly control the time evolution of the fragmentation process, but not the minimal fragment length.

However, when $K_{B}$ becomes smaller than 1, fragmentation can occur almost everywhere on the fibres as a small curvature becomes sufficient to break a fibre. The ensuing fragmentation process is therefore different than the one for $K_{B} \geq 1$. As the curvature fluctuations for fibres shorter than $L=1$ are limited (see Fig. 3(c)), fragmentation in that regime approximately stops when the typical curvature $\langle K\rangle^{\star}$ of the fibre is smaller than the critical one $K_{B}$. As $\langle K\rangle^{\star}$ scales with $L^{3}$ for fibres shorter than $L=1$ (see Eq. (10)), the typical fragment length obtained in this last regime may therefore be given by

$$
L^{\star} \sim K_{B}^{1 / 3},
$$

showing here a dependency on the brittleness of the fibres for very brittle fibres $\left(K_{B} \leq 1\right)$.

\section{STATISTICAL MODEL}

\section{A. Evolution equation}

To validate such description of the fragmentation process, and compare quantitatively the numerical and experimental results discussed above, we now derive a simple statistical model where the key ingredients discussed previously are 
implemented. Here, we only focus on the situation with $K_{B} \geq 1$, corresponding to the regime where the experiments and simulations have been performed. Fragmentation events are therefore rare and uncorrelated. We consider $n(L, t)$ the number of fibres of dimensionless length $L$ at time $t$. For a given breaking parameter $K_{B}$, the variation of $n(L, t)$ per unit time is given by the integro-differential equation

$$
\frac{\partial n(L, t)}{\partial t}=-n(L, t) p(L)+2 \int_{L}^{\infty} n\left(L^{\prime}, t\right) p\left(L^{\prime}\right) \gamma\left(L^{\prime}, L\right) \mathrm{d} L^{\prime}
$$

The first term on the right hand side of Eq. (18) corresponds to the loss of fibres of length $L$ that break with a probability $p(L)$ per unit time. The second term represents the number of fibres of different lengths $L^{\prime}>L$ that break into a fibre of length $L$ per unit time with a probability $p\left(L^{\prime}\right) \gamma\left(L^{\prime}, L\right)$. Here, $\gamma\left(L^{\prime}, L\right)$ represents the breaking location probability, i.e. the statistics on the breaking location along the fibre, as discussed previously. The factor 2 in front of the integral expresses binary fragmentation: there are indeed two symmetric ways to produce fibres of length $L$ with a fibre of length $L^{\prime}$. Equation (18) is rather classical and is relevant to several fragmentation processes [53-55]. As two fragmentation events are considered to be independent due to the small fibre inertia, no memory effect is present in this equation. It is also important to note that, here, the model time scale $t$ is not properly defined, as it cannot be related to any physical quantity. It can therefore be assimilated to an iteration time scale between different steps of the fragmentation process.

\section{B. Analytical solution for a simplified version of the probabilities}

The previous evolution equation (18) cannot be solved analytically with the form of the probabilities $p$ and $\gamma$ shown in Fig. 4. Before solving this equation numerically in Section IV C, we here obtain an analytical solution of Eq. (18) through some simplifications on the probabilities $p$ and $\gamma$. First, the breaking probability can be written in the form

$$
p\left(K_{B}, L\right)=p_{L_{0}} \zeta(L) L
$$

where $p_{L_{0}}$ is the local breaking probability (see Eq. (14)) of the longest fibres of length $L=L_{0}$, and $\zeta$ a function of the fibre length $L$. In order to approach the numerical evaluation of the breaking probability shown with solid lines in Fig. 3(c), one sets

$$
\zeta(L) \equiv 1 \text { for } L>L_{c} \quad \text { and } \quad \zeta(L) \equiv 0 \text { for } L<L_{c},
$$

where $L_{c}$ here stands for a critical length, from which the breaking probability $p$ decays drastically. Such approximation for the breaking probability $p$ with $L_{c}=2$ is shown with dashed lines in Fig. 3(c). In addition, the breaking location probability is approximated by $\gamma\left(L^{\prime}, L\right) \equiv 1 / L^{\prime}$, which means that the fragmentation events are equiprobable along the fibre in that case. This simplification is of course not valid for relatively short fibres, which break mainly in half, but is reasonable for very long fibres for which the contribution of the boundary layer is small. Such description is an over-simplification of what happens on the tips of the fibres, since the tips stay rigid and cannot break. Thus, this leads to an incorrect estimation of the presence of fragments shorter than $L=1$. Nevertheless, these assumptions might be useful to understand the flux of fragments larger than $L=1$. They transform Eq. (18) into

$$
\frac{\partial n_{>}}{\partial \tau}(L, \tau)=-n_{>}(L, \tau) L+2 \int_{L}^{\infty} n_{>}\left(L^{\prime}, \tau\right) \mathrm{d} L^{\prime}
$$

for $L>L_{c}$, and

$$
\frac{\partial n_{<}}{\partial \tau}(L, \tau)=2 \int_{L_{c}}^{\infty} n_{>}\left(L^{\prime}, \tau\right) \mathrm{d} L^{\prime},
$$

for $L<L_{c}$. The quantity $\tau=p_{L}^{0} t$ is here defined as a non-dimensional time scale. Equation (21) is a standard form of the so-called "fragmentation equation" [53, 54]. In Eq. (22), only the integral starting from $L_{c}$ remains, as fragments shorter than $L_{c}$ cannot break. It therefore represents the flux of fibres shorter than $L_{c}$ produced by the fragmentation of fibres longer than $L_{c}$. One first looks for an asymptotic solution of Eq. (21) as

$$
n_{>}(L, \tau)=f(\tau) e^{-L \tau}
$$

with $f$ a function of the non-dimensional time $\tau$. The time derivative of Eq. (23) injected into Eq. (21) leads to

$$
\frac{\dot{f}}{f}=\frac{2}{\tau}
$$



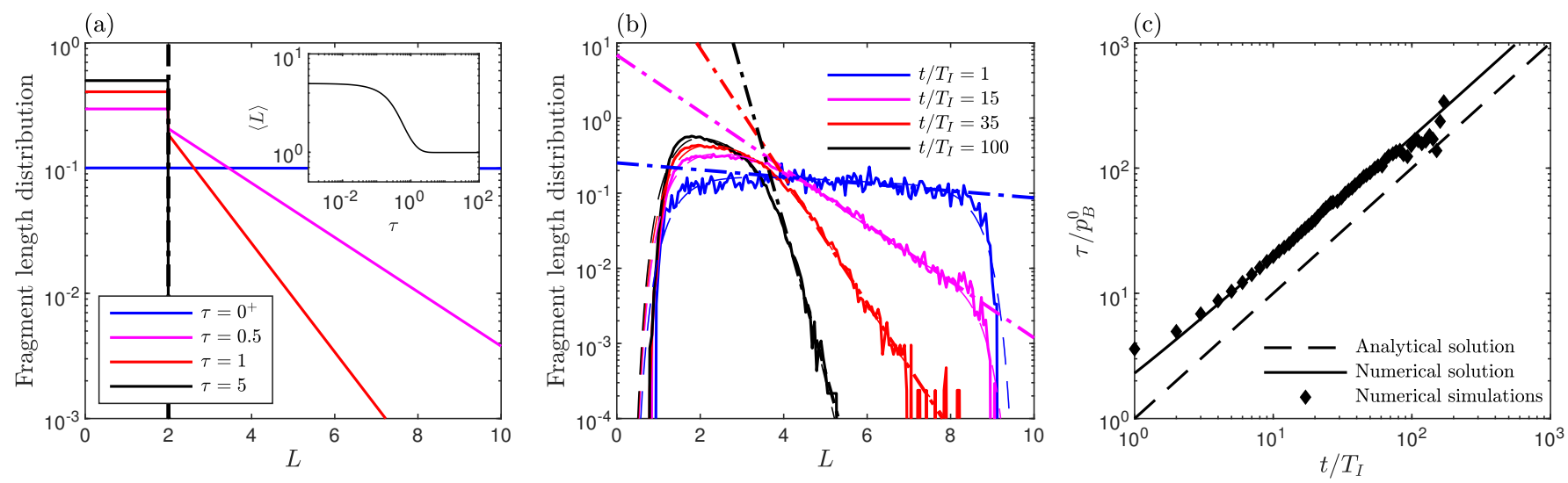

FIG. 5. Exponential tails of the length distributions. (a) Temporal evolution of the fragment length distribution obtained with the simplification of Eq. (18). The panel is in lin-log scales to emphasise on the exponential tail of the distribution. The vertical dashed dotted line shows $L=L_{c} \equiv 2$. The inset shows the temporal evolution of the mean fragment length $\langle L\rangle$. (b) Temporal evolution of the fragment length distribution with $K_{B}=2$, obtained in the numerical simulations (solid lines) and for the numerical solution of the full evolution equation (thin dashed lines, see Section IV C). The exponential fits of the tails are shown with dashed dotted thick lines. Note that this panel is also in lin-log scales to emphasise the tail of the distributions, contrary to Fig. 2(c) which is in log-log scales. (c) Correspondance between the theoretical time scale $\tau / p_{L}^{0}$ and the physical time scale $t / T_{I}$ for the numerical simulations and the numerical solution of the full evolution equation (see Section IVC).

and is independent of $L$. This gives a solution for $f$ as $\tau^{2}$ and for the number of fragments longer than $L_{c}$ as

$$
n_{>}(L, \tau) \sim \tau^{2} e^{-L \tau} .
$$

This number of fragments first increases with time $\tau$ before decaying and vanishing for $\tau \rightarrow+\infty$. To obtain the flux of fragment shorter than $L_{c}$, this solution for $n_{>}$is injected into Eq. (22), leading to

$$
\frac{\partial n_{<}}{\partial \tau}(L, \tau)=2 \int_{L_{c}}^{\infty} \tau^{2} e^{-L^{\prime} \tau} \mathrm{d} L^{\prime}=2 \tau e^{-L_{c} \tau} .
$$

Note that this flux is independent of the fibre length $L$, as the fragmentation events are equiprobable along fibres longer than $L_{c}$. The number of fragments shorter than $L_{c}$ is finally obtained by integrating Eq. (26) as

$$
\begin{aligned}
n_{<}(L, \tau) & =\int_{0}^{\tau} 2 \tau^{\prime} e^{-L_{c} \tau^{\prime}} \mathrm{d} \tau^{\prime}, \\
& =\frac{2}{L_{c}^{2}}\left(1-e^{-L_{c} \tau}\left(1+L_{c} \tau\right)\right) .
\end{aligned}
$$

The number of fragments $n_{<}$is also independent of the fibre length $L$ and saturates at $n_{<}=2 / L_{c}^{2}$ when $\tau \rightarrow+\infty$. The two distributions, $n_{<}$and $n_{>}$, are then gathered and normalised to obtain the fragment length distribution and the mean fragment length $\langle L\rangle$, both shown in Fig. 5(a). Note that with this piecewise approach, there is a strong discontinuity at $L=L_{c}$ when time increases. However, it allows us to discuss two main features of the fragment distributions also encountered in the simulations and experiments.

First, the relative fraction of fibres shorter than $L_{c}$ with respect to fibres longer than $L_{c}$, given by

$$
\frac{N_{<}(\tau)}{N_{>}(\tau)} \equiv \frac{\int_{0}^{L_{c}} n_{<}\left(L^{\prime}, \tau\right) \mathrm{d} L^{\prime}}{\int_{L_{c}}^{\infty} n_{>}\left(L^{\prime}, \tau\right) \mathrm{d} L^{\prime}}=\frac{2}{L_{c} \tau}\left(e^{L_{c} \tau}-\left(1+L_{c} \tau\right)\right),
$$

increases with time and finally diverges as $\tau^{-1} e^{L_{c} \tau}$ when $\tau \rightarrow+\infty$. It therefore shows an accumulation of fibres shorter than $L_{c}$ while the fibres longer than $L_{c}$ vanish after some time, as seen in Fig. $5(\mathrm{a})$ and also in Fig. 2 for the experiments and numerical simulations. However, such accumulation does not present any minimal fragment length in Fig. 5(a) as we remind that our simplified model cannot predict the length distribution of fragments shorter than $L=1$. By prohibiting any fibre to break too close to their tips, it is easy to imagine that the fragment length distributions shown in Fig. 5(a) would be truncated for $L<1$, thus exhibiting a minimal fragment length and a maximum. Using this 
simplified model, one can therefore decompose the role of the different ingredients in the fragmentation process: the fact that fibres do not break close to their tips gives a minimal fragment length around $L=1$, while the fact that fibres shorter than a critical length $L_{c}$ break much rarely leads to an accumulation of fragments in the range $L=1-L_{c}$, and to a maximum in the distribution.

Secondly, the simplified model predicts an exponential tail for the length distribution, as $e^{-L \tau}$ (see Eq. (25) and Fig. 5(a)). This particular feature is also verified in the fragment length distributions obtained with the numerical simulations, shown in Fig. 5(b). By fitting the tail of the numerical fragment length distributions in Fig. 5(b), the slope can be normalised by $p_{L}^{0}$ and plotted in Fig. $5(\mathrm{c})$ as a function of physical time scale $t / T_{I}$. This shows that the tail behaves well as $e^{-a L \tau}$, with $a \approx 2$ as a pre-factor. Such feature of the fragment length distribution arises from the fact that long fibres break almost everywhere, and indicates that the approximation on the breaking location probability $\gamma\left(L^{\prime}, L\right) \approx 1 / L^{\prime}$ is fully valid when one considers long fragments. Note that the tails of the experimental fragment length distributions also exhibit an exponential scaling with fragment length $L$ at different times, but this scaling is however not linear with time. This may be due to several reasons, further discussed in the next section.

\section{Comparison with experiments and simulations}

The analytical solution of a simplified version of Eq. (18) has allowed us to recover some key features of the fragment length distributions obtained both in the numerical simulations and experiments. However, in order to fully compare between the two, Eq. (18) has been solved numerically with Matlab using the trapezoidal rule, a first-order Euler temporal scheme, and the numerical evaluations of the breaking probability $p(L)$ and of the breaking location probability $\gamma\left(L^{\prime}, L\right)$ shown in Fig. 4.

The correspondance between the unknown fragmentation time scale in the model and the ones in the experiments or simulations is obtained through one single free parameter by comparing the temporal evolution of the mean fragment length $\langle L\rangle$, shown in Fig. 6. There is a good agreement between the model and the numerical simulations for the different breaking parameters (see Fig. 6(a)). For the experiments, it is necessary to account for the defects in the glass fibres that are randomly placed [46]. Indeed, they lead to a distribution of breaking parameters $K_{B}$ (see Appendix A 2), while the numerical simulations have only one breaking parameter $K_{B}$. As each breakup event is independent, one can therefore combine the model length distributions for different breaking parameters, and obtain the mean fragment length given by the dashed lines in Fig. 6(b). This matches well the experimental trend when several experiments have been performed (blue and red points), while a model with only one breaking parameter equal to the average of the distribution would lead to a faster time evolution like in Fig. 6(a). Note that when only one experimental point is available (purple or black point), the model is used to guide the eyes and to show a global evolution.

One can then compare the model fragment length distributions with the numerical and experimental ones. Across different time scales and breaking parameters, the agreement between the model and the numerical simulations in Figs. 2(c) (or Fig. 5(b)) and 2(d) is excellent. As expected, the tails of the fragment length distribution are exponential
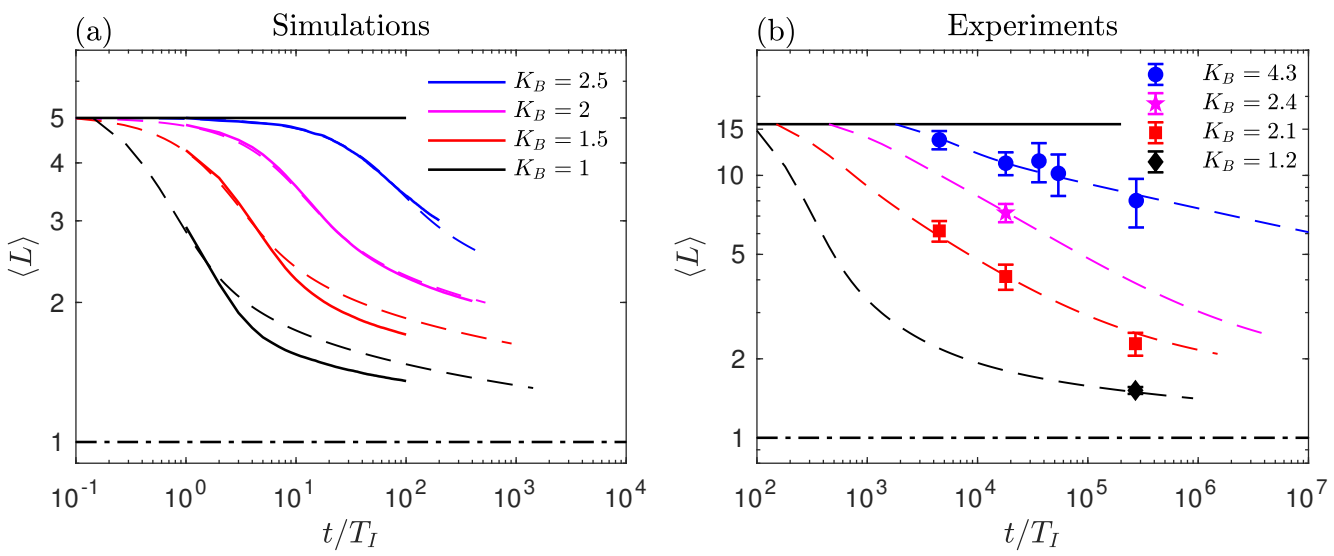

FIG. 6. Temporal evolution of mean fragment length $\langle L\rangle$. (a) Simulations (solid lines) and model (dashed lines). (b) Experiments (symbols) and model with a distribution of breaking parameters centred around the value indicated in the legend (dashed lines). The error bars on the experimental points are representative of the number of fragments collected for each experiment. In both panels, $L_{0} / 2$ and $L=1$ are indicated by the solid and dashed dotted horizontal black lines. 
and behaves well as $\exp (-a L \tau)$, as shown in Figs. 5(b) and (c). For short fragments, the small discrepancies are due to the overestimation of the curvature $\langle K\rangle$ close to the fibre tips by the fit in Fig. 3(a). This shows that the key ingredients implemented in the model are fully sufficient to describe the physics of the fragmentation process in the numerical simulations. The comparison with the experimental fragment length distributions in Fig. 2 is qualitatively good. The differences for very short and long fragments may be explained by the fact that fragmentation occurs in the vicinity of the propellers in the experiments. Indeed, using high-speed cameras and a schlieren technique [56], no fragmentation event has been observed in the bulk of the flow, while the regions close to the propellers are not optically accessible. Note that the low Stokes number of the fibres imposes that they follow the flow and therefore prevents them from touching the propellers directly (see Section II C). However, as large objects explore preferentially the regions close to the propellers [43], where the turbulence intensity is larger than in the rest of tank and the elastic length slightly shorter, long fibres may fragment more than what is predicted by the model and lead to a larger number of short fragments. While these considerations may be necessary to model the full temporal evolution and shape of the fragment length distributions (Fig. 2), a scenario purely based on rare fragmentation events in two equal pieces within the most turbulent regions of the flow fails to describe the fragment length distribution (see Appendix C). Thus, the local fibre-turbulence interactions, leading to the intrinsic role of the elastic length as a cut-off length, are the main contributors to the fragmentation process and control the global shape of the fragment length distribution.

\section{APPLICATION TO PLASTIC FRAGMENTATION IN THE OCEAN}

As the fragmentation process in the laboratory is limited by the elastic length $\ell_{e}$, we now extend the discussion on the validity of our model to marine plastic fragmentation. Even though the laboratory model discussed here deals with brittle fibres, one can reasonably expect that the fragmentation scenario is similar for brittle objects with other shapes, such as stripes or surfaces. For these 2D or 3D objects, the physics and the scaling laws of the elastic length may differ. Nevertheless, it is expected that small objects do not deform and therefore do not break in turbulence. The fragmentation scenario of our laboratory model can therefore be applied to a large range of plastic debris in the ocean, providing that they have been weathered enough to become brittle.

In addition, when the debris have a typical size much larger in one direction than in the others, one can expect the fragmentation process in the ocean to be effectively limited by the elastic length $\ell_{e}$ shown in Eq. (1). Therefore, it is worth evaluating this length scale in the ocean in order to compare to the typical measured size of marine plastic debris [17-19]. Let us consider an elongated object with a Young modulus $E \approx 1$ GPa and a diameter $d \approx 50 \mu$ m. These values are rather classical for plastic materials, as plastic films for example [57, 58] which have a typical thickness of $50 \mu \mathrm{m}$. The turbulent flow near the ocean surface experienced by plastic debris is most of the time weakly turbulent, with turbulent dissipation rates of the order of $\varepsilon \approx 10^{-6}-10^{-3} \mathrm{~m}^{2} / \mathrm{s}^{3}$ caused by wind shear on the ocean surface [21]. However, wave breaking events can lead to intermittent but intense and localised turbulent flows [59, 60], enhancing the turbulent energy dissipation rate by several orders of magnitude [31, 32] leading to $\varepsilon \approx 10^{-1}-10^{2} \mathrm{~m}^{2} / \mathrm{s}^{3}$. These intense values of the turbulent dissipation rate leads to an estimation of $\ell_{e} \approx 2-5$ mm for typical plastic debris in oceans, close to the peak of the plastic size distribution in oceans [17-19]. It therefore shows that the cut-off mechanism in the fragmentation process investigated in the laboratory is compatible with what is observed in oceans for plastic fragmentation and can explain both the lack of small debris compared to an expected power law and the existence of a peak in the distribution. Such features can therefore be explained by arguments solely based on fluidstructure interactions and it is not necessary to introduce several sinks for small microplastics [17]. It is also important to note that the elastic length depends only on the bulk properties of the material (Young modulus and diameter), and not on other mechanical properties (tensile strength, extensibility) that are more affected by weathering [61, 62]. This length is thus independent of the debris brittleness (here $K_{B}$ ), which plays only a role in the temporal evolution of the process. As a consequence, enhanced degradable plastics [57, 61, 63] may also accumulate at a millimetric size, like standard plastic debris, and therefore represent similar hazards through ingestion for example. However, when $K_{B}$ becomes smaller than 1, the typical fragment length starts to depend on the fibre brittleness, as shown in Eq. (17). Such scaling for $K_{B} \leq 1$ can explain why the fragment size distributions for plastic debris in oceans [17-19] are not sharply truncated at small scales, contrary to the fragment length distributions shown in this study for which the minimal length is independent of the brittleness of the fibres as $K_{B} \geq 1$.

The estimation of the elastic length shows that fragmentation events mainly occur in highly turbulent environments encountered by the plastic debris, i.e. during wave breaking events. Predicting the complete temporal evolution and shape of the fragment length distribution may therefore involve models based on rare events [64] as in Appendix C, coupled with a thorough understanding of the highly non-linear effect of the breaking parameter $K_{B}$, which is timedependent in the ocean due to the weathering of plastic debris. In addition, the existence of oceanic nanoplastic debris reported recently $[65,66]$ cannot be explained by the fragmentation process described in this study. Indeed, such debris are much smaller than the Kolmogorov scale while the fibres considered here are longer than this scale. 
Other fragmentation mechanisms have to be considered below the Kolmogorov length scale, such as stretching or compression, leading to different length distributions [67]. It is however our hope that the physical considerations in this paper about the fragmentation of flexible and brittle objects in turbulent flow will help to fill a gap in knowledge [14] on plastic fragmentation in oceans.

\section{ACKNOWLEDGMENTS}

This work was carried out in the framework of FlexFiT Project (ANR-17-CE30-0005-01) funded by the French National Research Agency (ANR). Centre de Calcul Intensif d'Aix-Marseille is acknowledged for granting access to its high performance computing resources. The authors particularly thank Dominique Font, from Owens Corning company, for freely providing the glass fibres used in this study.

\section{Appendix A: Mechanical properties of the fibres}

\section{Young modulus}

The Young modulus $E$ has been obtained by measuring the deflection $\delta$ from the horizontality for fibres placed at the edge of an horizontal plate. With $\ell$ the length of the fibre out of the plate, the deflection under its own weight is given using standard beam theory by

$$
\delta=\frac{2 \rho_{p} g \ell^{4}}{E d^{2}}
$$

where $\rho_{p} \simeq 2500 \mathrm{~kg} / \mathrm{m}^{3}$ is the tabulated glass density and $g$ the gravity acceleration. The deflection $\delta$ as a function of the length $\ell$ is shown in Fig. 7(a), in log-log scales. These measurements have been performed for raw and heat-treated fibres, as shown by the different colors and symbols. As expected from Eq. (A1), a power law $\delta \propto \ell^{4}$ is clearly visible for all data and shown by the dashed line. This gives a measure of the Young modulus of $E=41 \mathrm{GPa}$, independently of the nature of the fibres (raw or heat-treated).

\section{Estimation of the critical curvature}

Experimentally, the critical curvature $\kappa_{B}$ where fragmentation occurs has been estimated through measurements of the tensile strength $\sigma_{B}$ of the fibres (see Eq. (7)). The tensile strength of the glass fibres has been measured by pulling on the fibres at constant speed. One tip was glued to a fixed stage while the other was glued to a moving
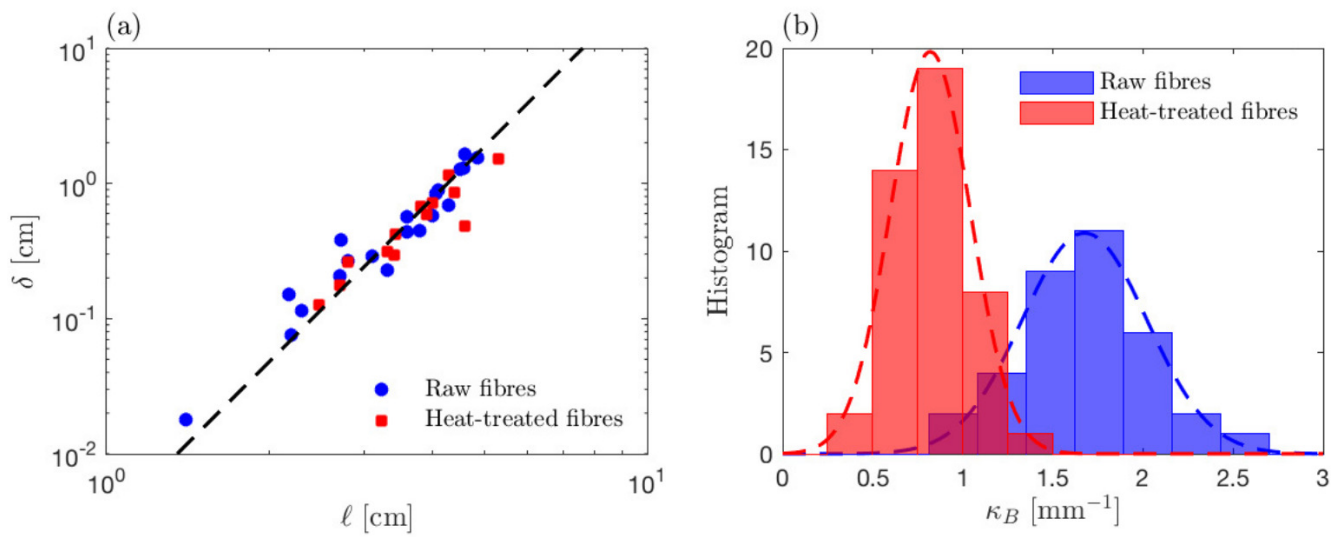

FIG. 7. Mechanical properties of the glass fibres used in this study. (a) Young modulus measurements: Vertical deflection $\delta$ from the horizontal of the tip of a fibre as a function of the length $\ell$. The dashed line shows the power law $\delta \propto \ell^{4}$ (see Eq. (A1)). (b) Histograms of the critical curvature $\kappa_{B}$ for raw fibres (blue) and heat-treated fibres (red). The dashed lines show Gaussian fits of the two histograms. 
stage, equipped with a force sensor. For each fibre, the force at break is measured and normalized by the section of the fibre to obtain the tensile strength. About 50 fibres have been tested for each batch, i.e. for raw and heat-treated fibres. The pulling speed has been varied within the range $10^{-2}-10 \mathrm{~mm} / \mathrm{s}$ without any effect on the measured tensile strength.

The statistics for the critical curvature $\kappa_{B}$ using Eq. (7) are shown in Fig. 7(b). They exhibit large distributions, typical of brittle materials where the tensile strength is governed by defects $[45,46,50]$. The dispersion of the critical curvature thus reflects the dispersion of the strength of the defects. The empirical Gaussian fits of these histograms allow us to determine the means and the variances of the critical curvature histograms. The critical curvature of the raw fibres has been measured to be in average about $\kappa_{B}=1.68 \pm 0.35 \mathrm{~mm}^{-1}$ while that of the heat-treated fibres is reduced by a factor of two in average, with $\kappa_{B}=0.82 \pm 0.22 \mathrm{~mm}^{-1}$. In the main body of the paper, the mean values are used in the different figures and in Table I, while the Gaussian fits are used for combining the different fragment length distributions of the model into one distribution, representative of the experiments and shown in Figs. 2(a) and (b), and in Fig. 6(b).

\section{Appendix B: Sensitivity to the initial fibre length $L_{0}$}

Fragmentation processes in the experiments, numerical simulations or model start with different initial fibre length $L_{0} \equiv \ell_{0} / \ell_{e}$. Indeed, experiments have been performed with $\ell_{0}=50$ or $80 \mathrm{~mm}$ and with two different elastic lengths. This leads to different $L_{0}$, from 19.2 to 53.3. In the numerics, all the simulations have been performed with $L_{0}=10$, which is also the integral scale of the flow. In the model, $L_{0}$ is a free parameter and has been set to 10 to compare with the numerical simulations and to 30.8 to compare with the experiments. It is however relevant to consider these data all together. Indeed, after a sufficient time, all the fragment length distributions with different initial fibre length $L_{0}$ collapse onto the same curve. This is shown in Fig. 8 for experiments and the model. The fragmentation length distributions are similar for experiments (panel (a)) and undistinguishable for the model (panel (b)). The time evolution of the mean fragment length $\langle L\rangle$, shown in the inset in Fig. 8(b), demonstrates that the evolution of the mean fragment length is different at early times but becomes independent of the initial fibre length $L_{0}$ for long time scales. Indeed, as the fragmentation process is governed by the elastic length that acts as a cut-off length, i.e. a minimal fragment length, the physics of the fragmentation process at long time scale or for short fragments is similar and does not depend on the fragment process of the long fragments, providing that $L_{0}$ is significantly larger than 1 . It also shows that it is relevant to compare and describe fragmentation processes that have different initial fibre lengths, as we do in the main body of the paper for experiments $\left(L_{0} \in[19.2-53.3]\right)$ and numerical simulations $\left(L_{0}=10\right)$.
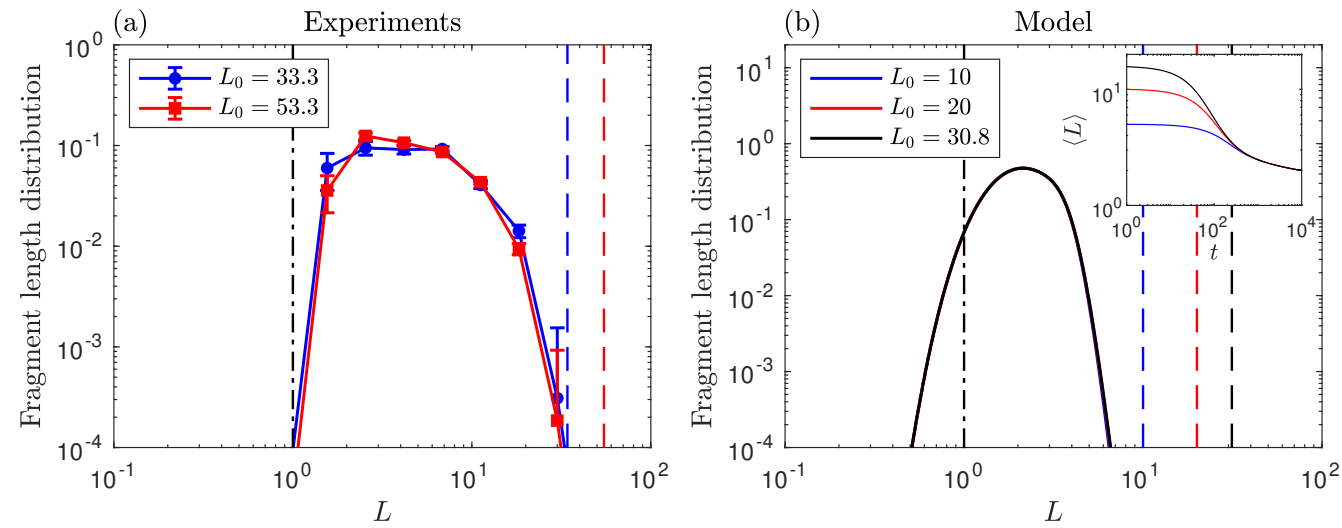

FIG. 8. Fragment length distributions with different initial fibre lengths $L_{0}$. (a) Experiments for $K_{B}=2.4$ at $t / T_{I}=18 \times 10^{3}$. The errorbars correspond to \pm 4 fragments. (b) Model for $K_{B}=2$ at $t=10^{3}$. The inset represents the time evolution of the mean fragment length for 3 different initial fibre lengths $L_{0}$. The colors correspond to different initial fibre lengths $L_{0}$, given in the legends and represented by vertical dashed lines in both panels. In both panels, the vertical dashed dotted lines indicate $L=1$. 


\section{Appendix C: Model based on rare fragmentation events in two equal pieces within highly turbulent regions}

The model presented above assumes that the fibres explore a large phase space of deformed configurations at each time step. This corresponds well to the dynamics observed in the numerical simulations as the fragmentation occurs rapidly. In the experiments, however, fragmentation events occur in the vicinity of the propellers, which are highly turbulent regions. It is therefore necessary to modulate the breaking probability of the fibres depending on their length, as short fibres explore similarly the whole tank while long fibres spend more time close to the propellers (see the discussion in the main body of the paper). Here we discuss another model, purely based on rare fragmentation events in the highest turbulent regions of the flow. In this model, we consider that the fragment length distribution is caused by the different histories of different fibres, because the time scale $T$ between two fragmentation events is very long. We consider that each fibre roughly breaks in two equal pieces after each fragmentation event, i.e. that after $k$ breakups, we have $L=2^{-k} L_{0}$. The fragment length distribution thus depends directly on the distribution of $k$ itself. The probability $P$ that exactly $k$ events occurred at time $t$ is given by

$$
P(k, t)=\frac{(t / T)^{k} \exp (-t / T)}{\Gamma(k+1)},
$$

where $\Gamma(k+1)=k \Gamma(k)=k$ !. Equation (C1) shows an exponential decay of the probability $P$ with time $T$, typical for large deviation theories $[64,68]$. The fragment length distribution, proportional to $n(L, t)$, is therefore given by $n(L, t) \mathrm{d} L=P(k, t) \mathrm{d} k$, with

$$
k=\frac{\ln \left(L_{0} / L\right)}{\ln 2} \text { and } \frac{\mathrm{d} L}{\mathrm{~d} k}=L
$$

This leads to

$$
n(L, t) \sim \frac{1}{L} \frac{(t / T)^{\ln \left(L_{0} / L\right)} \exp (-t / T)}{\Gamma\left(1+\ln \left(L_{0} / L\right)\right)} .
$$

One can then incorporate the breakage slow-down in the form of the divergence of $T$ for $L \rightarrow 1$ as

$$
T=\frac{T_{0}}{1-\exp (-(L-1))},
$$

with $T_{0}$ the mean time scale between two fragmentation events for long fibres, assumed to be a function of the breaking parameter $K_{B}$. Such divergence introduces the fact that long fibres break more easily than short ones, because they are more distorted by the flow and reach more often the critical curvature, but also because long fibres preferentially explore highly turbulent regions where fragmentation events occur. This naturally explains that it takes an infinite time to obtain fragments of length $L=1$ or shorter. Therefore, the corresponding fibres are inexistant in the flow
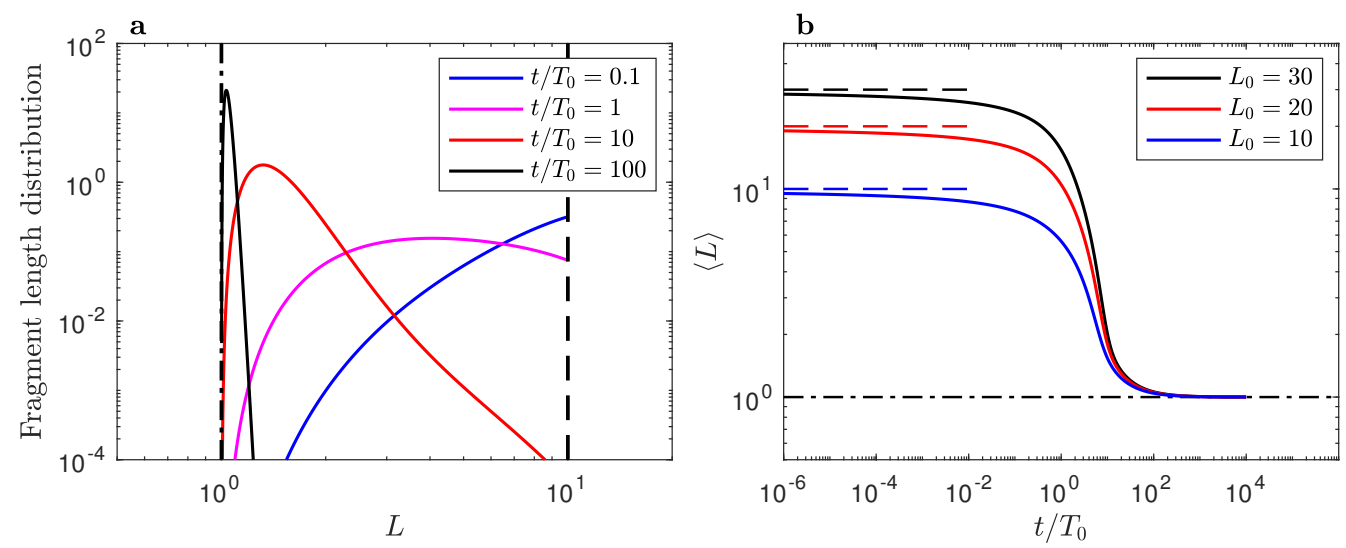

FIG. 9. Results obtained with the model based on rare fragmentation events in highly turbulent regions. (a) Time evolution of the fragment length distribution for $L_{0}=10$. The vertical dashed line shows the initial length while the vertical dashed dotted line indicates $L=1$. (b) Time evolution of the mean fragment length $\langle L\rangle$, for 3 different initial fibre lengths $L_{0}$. The horizontal dashed lines show the initial length with corresponding colors and the horizontal dashed dotted line indicates $L=1$. 

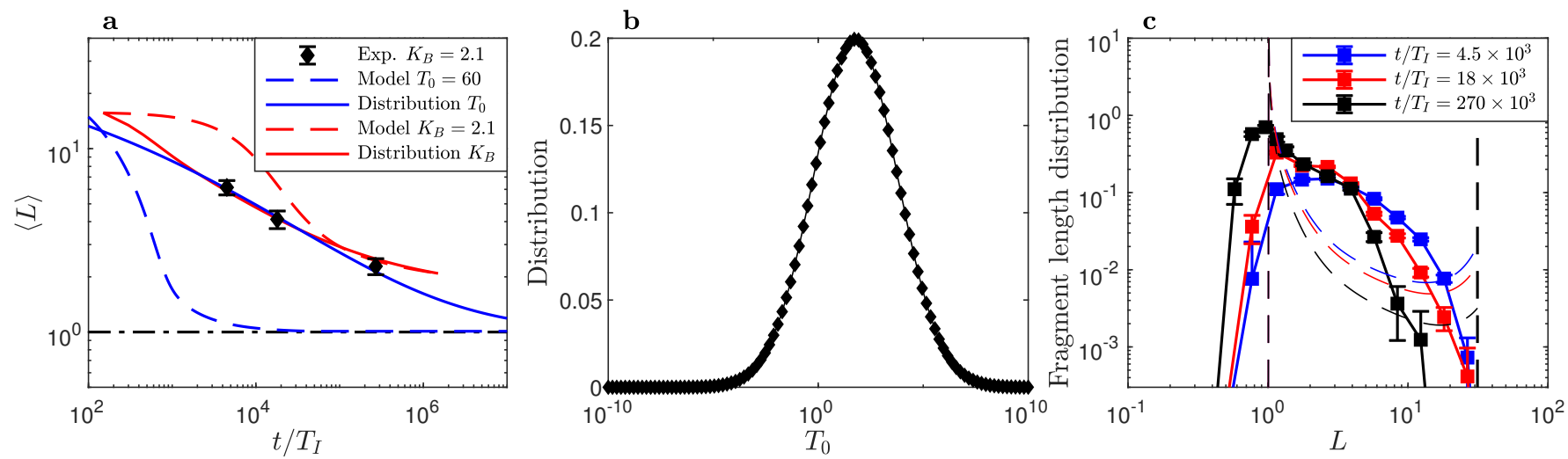

FIG. 10. Comparison with the experiments. (a) Temporal evolution of the mean fragment length $\langle L\rangle$ for experiments, and for the two models: the one based on the rare fragmentation events (blue) and the statistical one (red). This last model is mainly discussed in the main body of the paper (see Section IV C and Fig. 6(b)) and is here shown for a global comparison. For each model, the dashed line corresponds to the results with a single parameter $\left(T_{0}\right.$ or $\left.K_{B}\right)$ while the solid line represents the result for a distribution of the parameter considered. The horizontal dashed dotted line indicates $L=1$. (b) Distribution of the mean time scale $T_{0}$ between two fragmentation events for long fibres that allows us to match the temporal evolution of the experimental mean fragment length in panel (a). (c) Time evolution of the laboratory fragment length distribution for a fixed breaking parameter $K_{B}=2.1$, with error bars indicating \pm 4 fragments. With corresponding colors, the thin dashed lines represent the model discussed in this section. The vertical dashed line indicates $L_{0}$ for the model.

and the fragment length distribution has a minimal fragment length of $L=1$ and a maximum at long time scales around this value. Typical fragment length distributions are shown in Fig. 9(a). Compared to the fragment length distributions obtained with the statistical model or in the experiments and simulations, the shape of the distributions is here different at short time scales but is recovered for long time scales. The insensitivity of the length distributions to the initial fibre length $L_{0}$ is also recovered, as shown by the time evolution of the mean fragment length in Fig. 9(b).

In order to compare with the experiments, we again need to match the temporal evolution of the mean fragment length $\langle L\rangle$ in the experiments and in the model to find the correspondance between the model and the experimental time scales. Such matching is only possible when using a distribution of $T_{0}$, similarly to what has been done in the main body of the paper by using a distribution of breaking parameters $K_{B}$. Indeed, the temporal evolution of the mean fragment length for a single $T_{0}$ is not able to match the experimental time evolution, as shown by the blue dashed line in Fig. 10(a). However, choosing such distribution is rather arbitrary as the connection between the breaking parameter $K_{B}$ and the mean time scale $T_{0}$ between two fragmentation events for long fibres is not explicit. By testing several Gaussian distributions for $T_{0}$ in log-scale, we are able to obtain one distribution, represented in Fig. 10(b), for which the temporal evolution of the model mean fragment length matches well the experimental one (see blue solid line in Fig. 10(a)). Using such temporal evolution, it is however not possible to discriminate between this model and the statistical one discussed in the main body of the paper, as both fall within the experimental errorbars. It is therefore necessary to examine the fragment length distributions given by the model discussed in this section, and shown in Fig. 10(c). However, the results are clearly incompatible with the experimental length distributions, contrary to the statistical fragmentation model described in the main body of the paper (see Fig. 2). It therefore shows that, despite the fact that it is necessary to take into account the particularities of the experiments to explain the complete shape of the length distributions, the fragmentation process based on the cut-off mechanism originating from the fluid-fibre interactions is responsible for the most significant part of the fragment length distribution. If applicable, the effect of the presence of the fibres in highly turbulent environments susceptible to break them has then to be taken into account in addition to this first mechanism, to predict precisely the time evolution and shape of the length distribution.

[1] J. R. Jambeck, R. Geyer, C. Wilcox, T. R. Siegler, M. Perryman, A. Andrady, R. Narayan, and K. L. Law, "Plastic waste inputs from land into the ocean," Science 347, 768-771 (2015).

[2] R. Geyer, J. R. Jambeck, and K. L. Law, "Production, use, and fate of all plastics ever made," Sci. Adv. 3 (2017).

[3] A. L. Andrady, "The plastic in microplastics: A review," Mar. Pollut. Bull. 119, 12-22 (2017).

[4] PlasticsEurope, Plastics - the Facts 2018: An analysis of European plastics production, demand and waste data, Tech. Rep. 
(Plastics Europe, 2018).

[5] M. Filella, "Questions of size and numbers in environmental research on microplastics: methodological and conceptual aspects," Environ. Chem. 12, 527-538 (2015).

[6] A. L. Andrady, "Microplastics in the marine environment," Mar. Pollut. Bull. 62, 1596-1605 (2011).

[7] M. Eriksen, L. C. M. Lebreton, H. S. Carson, M. Thiel, C. J. Moore, J. C. Borerro, F. Galgani, P. G. Ryan, and J. Reisser, "Plastic pollution in the world's oceans: More than 5 trillion plastic pieces weighing over 250,000 tons afloat at sea," PLoS ONE 9, e111913 (2014).

[8] A. ter Halle, L. Ladirat, X. Gendre, D. Goudouneche, C. Pusineri, C. Routaboul, C. Tenailleau, B. Duployer, and E. Perez, "Understanding the Fragmentation Pattern of Marine Plastic Debris," Environ. Sci. Technol. 50, 5668-5675 (2016).

[9] L. A. Amaral-Zettler, E. R. Zettler, and T. J. Mincer, "Ecology of the plastisphere," Nat. Rev. Microbiol. 18, 139-151 (2020).

[10] E. R. Zettler, T. J. Mincer, and L. A. Amaral-Zettler, "Life in the "Plastisphere": Microbial Communities on Plastic Marine Debris," Environ. Sci. Technol. 47, 7137-7146 (2013).

[11] D. K. A. Barnes, F. Galgani, R. C. Thompson, and M. Barlaz, "Accumulation and fragmentation of plastic debris in global environments," Phil. Trans. R. Soc. B 364, 1985-1998 (2009).

[12] E. van Sebille, C. Wilcox, L. Lebreton, N. Maximenko, B. D. Hardesty, J. A. van Freneker, M. Eriksen, D. Siegel, F. Galgani, and K. L. Law, "A global inventory of small floating plastic debris," Environ. Res. Lett. 10 (2015).

[13] N. Schmidt, D. Thibault, F. Galgani, A. Paluselli, and R. Sempéré, "Occurrence of microplastics in surface waters of the Gulf of Lion (NW Mediterranean Sea)," Prog. Oceanogr. 163, 214-220 (2018).

[14] P. Notten, Addressing Marine Plastics : A Systemic Approach - Recommendations for Action., Tech. Rep. (United Nations Environment Programme, Nairobi, Kenya, 2019).

[15] G. Timár, J. Blömer, F. Kun, and H. J. Herrmann, "New universality class for the fragmentation of plastic materials," Phys. Rev. Lett. 104 (2010).

[16] H. Kishimura, D. Noguchi, W. Preechasupanya, and H. Matsumoto, "Impact fragmentation of polyurethane and polypropylene cylinder," Physica A 392, 5574-5580 (2013).

[17] A. Cózar, F. Echevarría, J. I. González-Gordillo, X. Irigoien, B. Úbeda, S. Hernández-León, Á. T. Palma, S. Navarro, J. García-de Lomas, A. Ruiz, M. L. Fernández-de Puelles, and C. M. Duarte, "Plastic debris in the open ocean," Proc. Natl. Acad. Sci. USA 111, 10239-10244 (2014).

[18] A. Cózar, M. Sanz-Martín, E. Martí, J. I. González-Gordillo, B. Ubeda, J. Á. Gálvez, X. Irigoien, and C. M. Duarte, "Plastic Accumulation in the Mediterranean Sea," PLoS ONE 10, e0121762 (2015).

[19] A. Cózar, E. Martí, C. M. Duarte, J. García-de Lomas, E. van Sebille, T. J. Ballatore, V. M. Eguíluz, J. I. GonzálezGordillo, M. L. Pedrotti, F. Echevarría, R. Troublè, and X. Irigoien, "The Arctic Ocean as a dead end for floating plastics in the North Atlantic branch of the Thermohaline Circulation," Sci. Adv. 3 (2017).

[20] N. Vandenberghe and E. Villermaux, "Geometry and fragmentation of soft brittle impacted bodies," Soft Matter 9, 81628176 (2013).

[21] E. A. D'Asaro, "Turbulence in the Upper-Ocean Mixed Layer," Annu. Rev. Mar. Sci. 6, 101-115 (2014).

[22] D. H. Bache, "Floc rupture and turbulence: a framework for analysis," Chem. Eng. Sci. 59, 2521-2534 (2004).

[23] M. U. Bäbler, M. Morbidelli, and J. Baldyga, "Modelling the breakup of solid aggregates in turbulent flows," J. Fluid Mech. 612, 261-289 (2008).

[24] M. U. Bäbler, L. Biferale, L. Brandt, U. Feudel, K. Guseva, A. S. Lanotte, C. Marchioli, F. Picano, G. Sardina, A. Soldati, and F. Toschi, "Numerical simulations of aggregate breakup in bounded and unbounded turbulent flows," J. Fluid Mech. 766, 104-128 (2015).

[25] C. Marchioli and A. Soldati, "Turbulent breakage of ductile aggregates," Phys. Rev. E 91 (2015).

[26] D. Saha, M. U. Babler, M. Holzner, M. Soos, B. Lüthi, A. Liberzon, and W. Kinzelbach, "Breakup of Finite-Size Colloidal Aggregates in Turbulent Flow Investigated by Three-Dimensional (3D) Particle Tracking Velocimetry," Langmuir 32, 55-65 (2016).

[27] B. Oyegbile, P. Ay, and S. Narra, "Flocculation kinetics and hydrodynamic interactions in natural and engineered flow systems: A review," Environ. Eng. Res. 21, 1-14 (2016).

[28] H. M. De La Rosa Zambrano, G. Verhille, and P. Le Gal, "Fragmentation of magnetic particle aggregates in turbulence," Phys. Rev. Fluids 3 (2018).

[29] S. A. Vanapalli, S. L. Ceccio, and M. J. Solomon, "Universal scaling for polymer chain scission in turbulence," Proc. Natl. Acad. Sci. U.S.A. 103, 16660-16665 (2006).

[30] D. Vincenzi, T. Watanabe, S. S. Ray, and J. R. Picardo, "Polymer scission in turbulent flows," J. Fluid Mech. in press (2020).

[31] J. R. Gemmrich and D. M. Farmer, "Near-Surface Turbulence in the Presence of Breaking Waves," J. Phys. Oceanogr. 34, 1067-1086 (2004).

[32] J. R. Gemmrich, "Strong Turbulence in the Wave Crest Region," J. Phys. Oceanogr. 40, 583-595 (2010).

[33] T. R. Powers, "Dynamics of filaments and membranes in a viscous fluid," Rev. Mod. Phys. 82, 1607-1631 (2010).

[34] C. Brouzet, G. Verhille, and P. Le Gal, "Flexible Fiber in a Turbulent Flow: A Macroscopic Polymer," Phys. Rev. Lett. $112(2014)$.

[35] A. Gay, B. Favier, and G. Verhille, "Characterisation of flexible fibre deformations in turbulence," EPL 123 (2018).

[36] M. E. Rosti, A. A. Banaei, L. Brandt, and A. Mazzino, "Flexible Fiber Reveals the Two-Point Statistical Properties of Turbulence," Phys. Rev. Lett. 121 (2018). 
[37] S. Allende, C. Henry, and J. Bec, "Stretching and Buckling of Small Elastic Fibers in Turbulence," Phys. Rev. Lett. 121 (2018).

[38] F. Ravelet, Bifurcations globales hydrodynamiques et magnétohydrodynamiques dans un écoulement de von Kármán turbulent, Ph.D. thesis, École Polytechnique, CEA (2005).

[39] B. Eckhardt and J. Schumacher, "Turbulence and passive scalar transport in a free-slip surface," Phys. Rev. E 64 (2001).

[40] G. Boffetta, J. Davoudi, B. Eckhardt, and J. Schumacher, "Lagrangian Tracers on a Surface Flow: The Role of Time Correlations," Phys. Rev. Lett. 93 (2004).

[41] S. Lovecchio, C. Marchioli, and A. Soldati, "Time persistence of floating-particle clusters in free-surface turbulence," Phys. Rev. E 88 (2013).

[42] S. Lovecchio, F. Zonta, and A. Soldati, "Upscale energy transfer and flow topology in free-surface turbulence," Phys. Rev. E 91 (2015).

[43] N. Machicoane, R. Zimmermann, L. Fiabane, M. Bourgoin, J.-F. Pinton, and R. Volk, "Large sphere motion in a nonhomogeneous turbulent flow," New J. Phys. 16 (2014).

[44] R. J. Kerekes, "Rheology of fibre suspensions in papermaking: An overview of recent research," Nord. Pulp Pap. Res. J. 21, 100-114 (2006).

[45] D. Sinclair, "A Bending Method for Measurement of the Tensile Strength and Young's Modulus of Glass Fibers," J. Appl. Phys. 21, 380-386 (1950).

[46] S. Feih, K. Manatpon, Z. Mathys, A. G. Gibson, and A. P. Mouritz, "Strength degradation of glass fibers at high temperatures," J. Mater. Sci. 44, 392-400 (2009).

[47] B. Herzhaft and É. Guazzelli, "Experimental study of the sedimentation of dilute and semi-dilute suspensions of fibres," J. Fluid Mech. 384, 133-158 (1999).

[48] É. Guazzelli and J. F. Morris, A Physical Introduction to Suspension Dynamics, Cambridge Texts in Applied Mathematics (Cambridge University Press, 2011).

[49] J. C. H. Fung, J. C. R. Hunt, N. A. Malik, and R. J. Perkins, "Kinematic simulation of homogeneous turbulence by unsteady random fourier modes," J. Fluid Mech. 236, 281-318 (1992).

[50] R. Gy, "Stress corrosion of silicate glass: a review," J. Non-Cryst. Solids 316, 1-11 (2003).

[51] S. M. Wiederhorn, "Influence of water vapor on crack propagation in soda-lime glass," J. Am. Ceram. Soc. 50, 407-414 (1967).

[52] L. Vanel, S. Ciliberto, P.-P. Cortet, and S. Santucci, "Time-dependent rupture and slow crack growth: elastic and viscoplastic dynamics," J. Phys. D: Appl. Phys. 42 (2009).

[53] E. W. Montroll and R. Simha, "Theory of Depolymerization of Long Chain Molecules," J. Chem. Phys. 8, 721-727 (1940).

[54] P. L. Krapivsky and E. Ben-Naim, "Scaling and multiscaling in models of fragmentation," Phys. Rev. E 50, 3502-3507 (1994).

[55] E. Villermaux, "Fragmentation versus Cohesion," J. Fluid Mech. 898, P1 (2020).

[56] G. S. Settles and M. J. Hargather, "A review of recent developments in schlieren and shadowgraph techniques," Meas. Sci. Technol. 28 (2017).

[57] A. L. Andrady, J. E. Pegram, and S. Nakatsuka, "Studies on Enhanced Degradable Plastics: II. Weathering of Enhanced Photodegradable Polyethylenes Under Marine and Freshwater Floating Exposure," J. Environ. Polym. Degrad. 1, 117-126 (1993).

[58] N. F. A. Biber, A. Foggo, and R. C. Thompson, "Characterising the deterioration of different plastics in air and seawater," Mar. Pollut. Bull. 141, 595-602 (2019).

[59] C. Garrett, M. Li, and D. M. Farmer, "The Connection between Bubble Size Spectra and Energy Dissipation Rates in the Upper Ocean," J. Phys. Oceanogr. 30, 2163-2171 (2000).

[60] G. B. Deane and M. D. Stokes, "Scale dependence of bubble creation mechanisms in breaking waves," Nature 418, 839-844 (2002).

[61] A. L. Andrady, J. E. Pegram, and S. Nakatsuka, "Studies on Enhanced Degradable Plastics: I. The Geographic Variability in Outdoor Lifetimes of Enhanced Photodegradable Polyethylenes," J. Environ. Polym. Degrad. 1, 31-43 (1993).

[62] F. Carrasco, P. Pagès, S. Pascual, and X. Colom, "Artificial aging of high-density polyethylene by ultraviolet irradiation," Eur. Polym. J. 37, 1457-1464 (2001).

[63] T. O'Brine and R. C. Thompson, "Degradation of plastic carrier bags in the marine environment," Mar. Pollut. Bull. 60, 2279-2283 (2010).

[64] H. Touchette, "The large deviation approach to statistical mechanics," Phys. Rep. 478, 1-69 (2009).

[65] J. Gigault, B. Pedrono, B. Maxit, and A. ter Halle, "Marine plastic litter: the unanalyzed nano-fraction," Environ. Sci.: Nano 3, 346-350 (2016).

[66] A. ter Halle, L. Jeanneau, M. Martignac, E. Jardé, B. Pedrono, L. Brach, and J. Gigault, "Nanoplastic in the North Atlantic Subtropical Gyre," Environ. Sci. Technol. 51, 13689-13697 (2017).

[67] S. Allende, C. Henry, and J. Bec, "Dynamics and fragmentation of small inextensible fibers in turbulence," Phil. Trans. R. Soc. A 378 (2020).

[68] M. Wilkinson, "Large Deviation Analysis of Rapid Onset of Rain Showers," Phys. Rev. Lett. 116 (2016). 